\title{
Use of Variational Techniques for the Estimation of Neutron Detection Efficiency
}

\author{
Sheng-chi Lin \\ J. C. Robinson \\ G. F. Flanagan
}

\section{OAK RIDGE NATIONAL LABORATORY}




\section{DISCLAIMER}

This report was prepared as an account of work sponsored by an agency of the United States Government. Neither the United States Government nor any agency Thereof, nor any of their employees, makes any warranty, express or implied, or assumes any legal liability or responsibility for the accuracy, completeness, or usefulness of any information, apparatus, product, or process disclosed, or represents that its use would not infringe privately owned rights. Reference herein to any specific commercial product, process, or service by trade name, trademark, manufacturer, or otherwise does not necessarily constitute or imply its endorsement, recommendation, or favoring by the United States Government or any agency thereof. The views and opinions of authors expressed herein do not necessarily state or reflect those of the United States Government or any agency thereof. 


\section{DISCLAIMER}

Portions of this document may be illegible in electronic image products. Images are produced from the best available original document. 
Printed in the United States of America. Available from National Technical Information Service

U.S. Department of Commerce

5285 Port Royal Road, Springfield, Virginia 22161

Price: Printed Copy \$6.50; Microfiche $\$ 2.25$

This report was prepared as an account of work sponsored by the United States Government. Neither the United States nor the Energy Research and Development Administration, nor any of their employees, nor any of their contractors, subcontractors, or their employees, makes any warranty, express or implied, or assumes any legal liability or responsibility for the accuracy, completeness or usefulness of any information, apparatus, product or process disclosed, or represents that its use would not infringe privately owned rights. 
ORNL/TM-5239

UC-79d - LMFBR Physics

Contract No. W-7405-eng-26

Neutron Physics Division

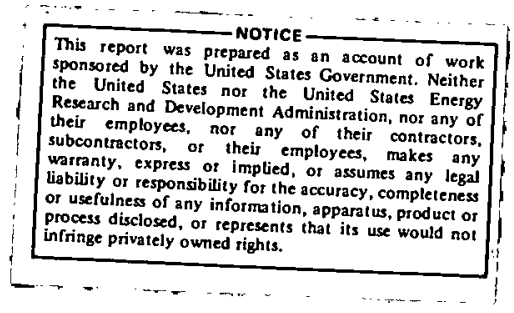

USE OF VARIATIONAL TECHNIQUES FOR THE ESTIMATION OF NEUTRON DETECTION EFFICIENCY ${ }^{\dagger}$

\author{
Sheng-chi Lin* J. C. Robinson* \\ G. F. Flanagan
}

FEBRUARY 1976

tSubmitted to the University of Tennessee by Sheng-chi Lin as a doctoral dissertation in the Department of Nuclear Engineering.

*Nuclear Engineering Dept., The University of Tennessee, Knoxvitle.

OAK RIDGE NATIONAL LABORATORY

Oak Ridge, Tennessee 37830

operated by

UNION CARBIDE CORPORATION

for the

ENERGY RESEARCH AND DEVELOPMENT ADMINISTRATION 
THIS PAGE

WAS INTENTIONALLY

LEFT BLANK 


\section{PREFACE AND ACKNOWLEDGMENTS}

This report describes work performed by Sheng-chi Lin in partial fulfillment of the requirements for a doctoral degree in the Nuclear Engineering Department of The University of Tennessee under the direction of his major professor, Dr. J. C. Robinson, and a staff member of ORNL's Neutron Physics Division, Dr. G. F. Flanagan. Mr. Lin received his support from the Chinese Government throughout his graduate studies at The University, and computer time was provided through the Neutron Physics Division by ERDA's Liquid Metal Fast Breeder Reactor Program.

The authors wish to express appreciation to the many persons who gave helpful assistance throughout this work, but particularly to D. L. Selby of ORNL. Thanks are also given to R. L. Childs, also of ORNL, for his permission to use his unpublished code VIP.

Special thanks are also due to Mrs. Juanita Rye, who typed the report. 
THIS PAGE

WAS INTENTIONALLY

LEFT BLANK 


\section{ABSTRACT}

The neutron detection efficiency is a parameter required in the measurement of reactivity by the modified source technique. The direct solution of the detection efficiency at a perturbed state is costly.

To solve for this, a particular variational functional, the Lewins' type variational functional, was presented in this study. The functional is a ratio of two other functionals, each dealing with a reaction rate. The evaluation of this particular functional was done by treating the numerator and the denominator functionals separately. This leads to three flux equations, one for forward flux, and two for adjoint fluxes. The advantages of this formulation over, and the equivalence of this formulation to, the conventional functional presented in the literature are described in detail.

The flexibility of the proposed functional was demonstrated by using it to estimate the detection efficiency with four different methods: variational interpolation, conventional variational, variational extrapolation, and multi-reference-state variational.

Results were demonstrated for one-dimensional and twodimensional problems. All results were compared with direct calculations. In all cases, the results show that the variational interpolational method and the multi-reference-state variational method are efficient and practically acceptable. 


\section{THIS PAGE}

\section{WAS INTENTIONALLY LEFT BLANK}


CHAPTER

PAGE

I. NATURE OF PROBLEM AND RELATED BACKGROUND ...... .1

Problem Statement ................

Conventional Variational Method for a Ratio

of Reaction Rates............... . 3

Objectives................ 7

II. MATHEMATICAL FORMULATION ............ 8

Introduction............... 8

Lewins' Variational Functional . ........ 8

The Equivalence Between the Lewins' Functional and

the Pọmaning's Functional . . . . . . . . 12

Extensions of the Lewins' Functional . . . . . . 15

Conventional Variational Method ........ 16

Variational Interpolational Method....... 16

Variational Extrapolational Method . . . . . 18

Multi-Reference-State Variational Method . . . . 18

III. COMPUTATIONAL PROCEDURES . . . . . . . . . 23

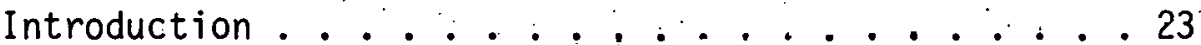

The Numerical Form of the Lewins' Functional . . . 23

Calculational Procedurés .................. 31

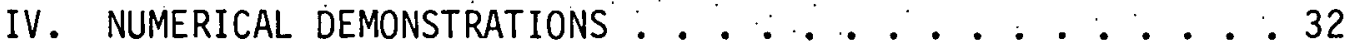

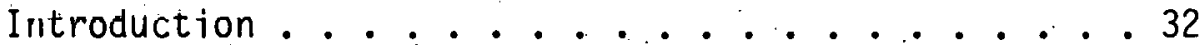

One-Dimensional Calculations ......... 33 
Description . . . . . . . . . 33

Reference States and Reference-Flux Calculation . . 36

Variational Calculational Methodology ...... 38

Results . . . . . . . . . . 39

Discussion of Results . . . . . . . . . 39

Two-Dimensional Calculations ........ . 55

Description .............. 55

Reference States and Flux Calculations .... 57

Variational Calculational Methodology..... . . 60

Results of Two-Dimensional Calculations . . . . 60

Discussion of Results ........... 65

V. CONCLUSIONS AND RECOMMENDATIONS .......... 67

Conclusions ....................... 67

Recommendations for Further Study . . ..... 68

BIBLIOGRAPHY . . . . . . . . . . . . . . 70

APPENDIXES . . . . . . . . . . . . 75

A. THE VARIATIONAL PRINCIPLE FOR A RATIO $\ldots \ldots \ldots$

B. TAYLOR'S SERIES EXPANSION OF $\theta^{*} \ldots \ldots \ldots$

C. SUPERPOSITION PRINCIPLE FOR A DIFFERENTIAL EQUATION . . . 82

D. DISCRETE FORM OF THE VARIATIONAL FUNCTIONAL ,FOR

SLAB GEOMETRY . . . . . . . . . . . . . . 84

E. CALCULATION OF $<\bar{\theta}^{\star}, \mathrm{S}>$ FOR ONE-DIMENSIONAL PROBLEM . . . 87

Introduction .................... 87

Program Description . . . . . . . . . 87 
Input Cards .................. 88

Listing of the Code . . . . . . . . . 88

F. CALCULATION OF $\langle\bar{\theta} *, S>$ FOR TWO-DIMENSIONAL PROBLEM . . . 91

Introduction ................... 91

Program Description ............. 91

Input Cards .................... 92

Listing of the Code ............. 92 
THIS PAGE

WAS INTENTIONALLY

LEFT BLANK 
TABLE

PAGE

1. Boundary Conditions for Forward and Adjoint Neutron Transport Equations .............. 25

2. States and Subcriticalities for One-Dimensional

Calculations ...................... 37

3. The Détection Efficiency of $U-235$ and He-3 Detector

at Each State by One-Dimensional Calculation . . . . 40

4. The Detection Efficiency of U-235 Detectoriby Conventional

Variational Method for One-Dimensional Core . . . . . 41

5. The Detection Efficiency of He-3 Detector by Conventional

Variationa T Method for One-Dimensional Core . . . . . 42

6. The Detection Efficiency of U-235 Detector by Variational.

Interpolation for One-Dimensional Core ....... 43

7. The Detection Efficiency of He-3 Detector by Variational

Interpolation for One-Dimensional Core . . . . . 45

8. The Detection Efficiency of U-235 Detector by Variational

Extrapolation for One-Dimensional Core . . . . . 47

9. The Detection Efficiency of He-3 Detector by Variational

Extrapolation for One-Dimensional Core ...... 49

10. The Detection Efficiency of U-235 Detector by Multi-

Reference-State Variational Method for One-

Dimensional Core ............... 50

11. The Detection Efficiency of He-3 Detector by Multi-

Reference-State Variational Method for One-Dimensional

core ........................ 51 
12. 14-Energy Group Structure and Fission Spectrum . . . . . 56

13. Buckling Cross Sections in Axial. Direction for Core Zones and Radial Reflector $\left(\mathrm{cm}^{-1}\right) \ldots \ldots$

14. Configurations of Reference States and Perturbed States for Two-Dimensional Calculations . . . . . . . 59

15. 14-Group Fission Cross-Section Collapsed from 50-Group

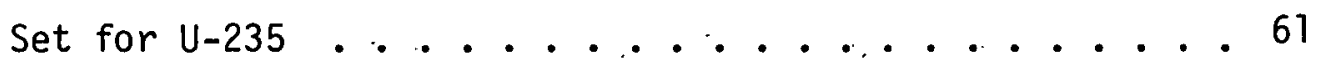

16. Neutron Detection Efficiency for Fission Chambers at $D_{1}$ and $\mathrm{D}_{3}$ Calculated by $\mathrm{DOT}$................... 62

17. Two-Dimensional Detection Efficiency Estimated by

Variational Interpolational Method .......... 63

18. Two-Dimensional Detection Efficiency Estimated by MultiReference-State Variational Method ......... 64 


\section{LIST OF FIGURES}

FIGURE

PAGE

1. Locations of Reference States and the Perturbed States for Various Variational Method . . . . . . . 17

2. Slab (half) Core Configuration for One-Dimensional Calculations ....................... 34

3. The EMC Core Configuration at Midpiane ......... 35 
THIS PAGE

WAS INTENTIONALLY

LEFT BLANK 


\section{NOMENCLATURE}

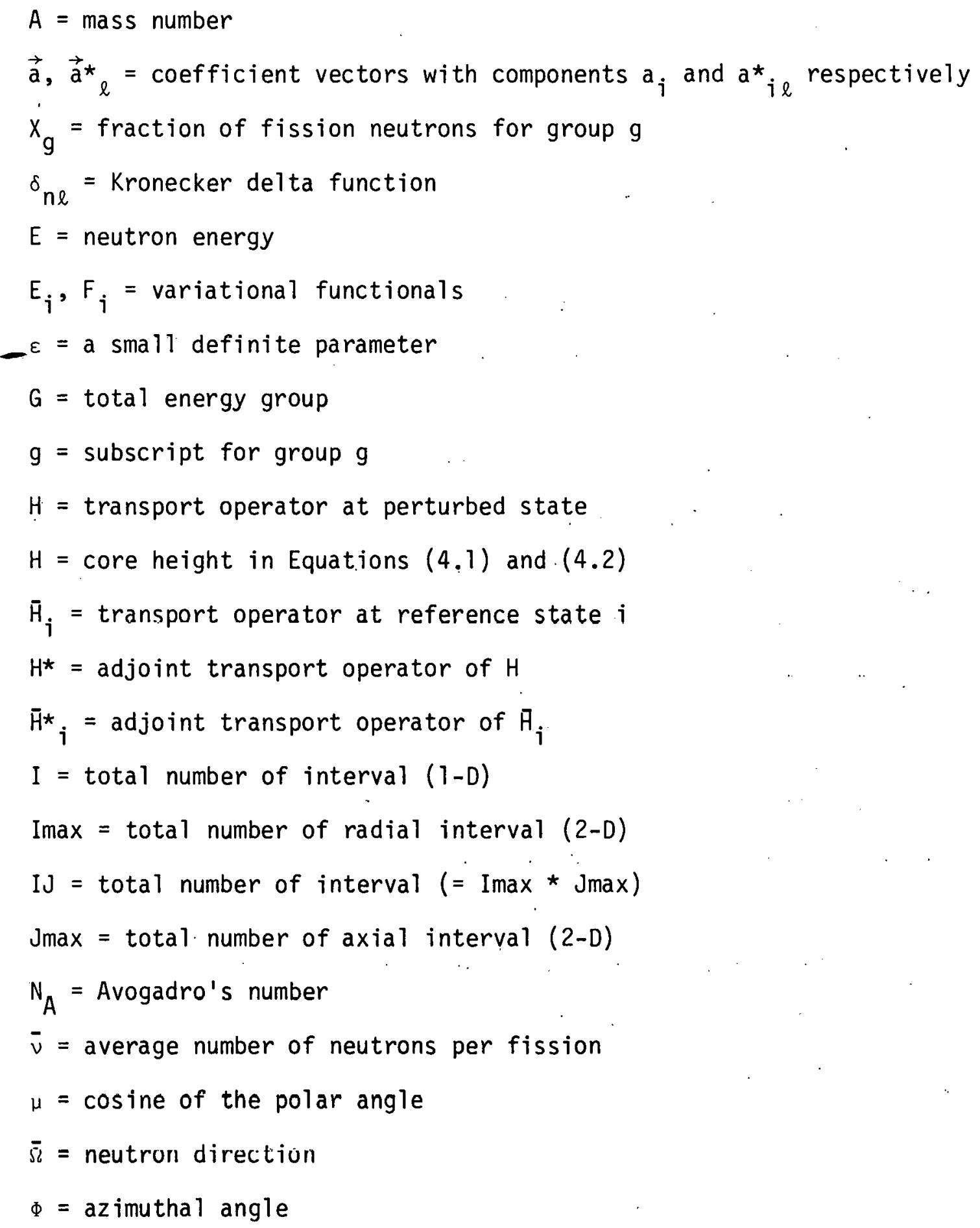


$\phi=$ neutron flux at perturbed state

$\bar{\phi}_{\dot{j}}=$ neutron flux at reference state $i$

$\theta^{*}=$ adjoint flux at perturbed state in Equation (2.3)

$\theta^{\star}{ }_{i}=$ adjoint flux in Equations (2.7) and (2.9), $i=1,2$

$\bar{\theta}^{\star}{ }_{j}=$ adjoint flux in Equations (2.11) and (2.12), $i=1,2$

$S, S=$ external neutron source of perturbed ànd reference states, respectively

$\Sigma=$ total macroscopic cross section

$\Sigma_{\mathrm{d}}=$ macroscopic detection cross section

$\Sigma_{f}=$ macroscopic fission cross section

$\Sigma_{S}=$ macroscopic scattering cross section

$\bar{\phi}_{\mathrm{gn}}^{\mathrm{K}}(\bar{r})=$ reference flux moment at position $\bar{r}$ of group $\mathrm{g}$

$\bar{\theta}^{\star}{ }_{i g n}^{K}(\bar{r})=$ adjoint reference flux moment at position $\bar{r}$ of group $g$,

$$
i=1,2
$$

$w=s l a b$ width

$W_{n}[\phi]=\frac{\left\langle\Sigma_{1} \phi\right\rangle}{\left\langle\Sigma_{2} \phi\right\rangle}, \Sigma_{1}$ and $\Sigma_{2}$ are two different cross-sections

$x=$ phase space in Equations (2.29) and (2.30)

$\Delta x, \Delta x_{i}=$ radial interval size at detector position and the $i^{\text {th }}$ interval, respectively

$\Delta y, \Delta y_{j}=$ axial interval size at detector position and the $j^{\text {th }}$ interval, respectively

$\mathrm{Y}_{\mathrm{n}}^{\mathrm{K}}(\bar{\Omega})=$ spherical harmonics 


\section{CHAPTER I}

\section{NATURE OF PROBLEM AND RELATED BACKGROUND}

\section{A. Problem Statement}

An important parameter in the Modified Source Multiplication formalism used in the measurement of reactor subcriticality is the neutron detection efficiency for fast or thermal power reactors. $(1,2,5,18,19,32)$

The neutron detection efficiency is defined ${ }^{(32)}$ as the number of neutron reactions in the detector per fission in the reactor core zone, i.e.;

$$
\begin{gathered}
W_{n}[\phi]=\frac{\int_{V_{d}} \delta_{E} \Sigma_{d}(\bar{r}, E) \phi(\bar{r}, E) d E d \bar{r}}{\int_{V_{C}} \int_{E} \Sigma_{f}(\bar{r}, E) \phi(\bar{r}, E) d E d \bar{r}} \\
=\frac{<\Sigma_{d} \phi>}{<\Sigma_{f} \phi>},
\end{gathered}
$$

where $\Sigma_{d}$ and $\Sigma_{f}$ are the macroscopic neutron cross sections for response in the detector and fission in the reactor core, respectively. $\phi$ is the neutron flux, and the $<$ represents the integration, or summation over phase space. $V_{d}$ and $V_{c}$ are the detector and core volume, respectively.

The calculation of $W_{n}$ is usually carried out $(5,12,14,19,33,43)$ by solving the time-independent neutron Boltzmann transport equation (4) for $\psi$, i.e., solve 


$$
\begin{aligned}
& \bar{\Omega} \cdot \nabla_{\phi}(\bar{r}, E, \bar{\Omega})+\sum(\bar{r}, E) \phi(\bar{r}, E, \bar{\Omega})= \\
& \frac{X(E)}{4 \pi} \int \delta \Sigma_{f^{\prime}}\left(\bar{r}, E^{-}\right) \phi\left(\bar{r}, E^{-}, \vec{\Omega}^{-}\right) \mathrm{dE}^{-} \mathrm{d}^{-} \bar{\Omega}^{-}+ \\
& \iint \Sigma_{S^{\prime}}\left(\bar{r}, E^{-}, \bar{\Omega}^{-} \rightarrow E^{\prime}, \bar{\Omega}\right) \phi\left(\bar{r}, E^{-}, \bar{\Omega}^{-}\right) \mathrm{dE}^{-} \mathrm{d} \bar{\Omega}^{-}+\mathrm{S}\left(\bar{r}, E^{-}, \bar{\Omega}\right),
\end{aligned}
$$

where the symbols used in Equation (1.2) have their conventional meaning as specified in reference 4. For convenience, we will express Equation (1.2) in operator form as:

$$
H \dot{\phi}=S,
$$

where

$$
H=\text { the transport operator for Equation (1.2); }
$$

and

$$
S=\text { the external neutron source.. }
$$

Once $\phi$ is known, Equation (1.1) is solved directly.

The difficulty with the direct computation of $w_{n}$ lies in the expensive computational effort required for the solution of Equation (1.2). A solution is required at each state it is desired to compute $w_{n}$.

We seek an acceptable approximate technique, a variational method, for the computation of $w_{n}$. The motivation for this approach is to avoid the repeated calculation of neutron flux by Equation (1.2). The conventional approach of the variational method for a parameter is described in detail in the following section. 
B. Conventional Variational Method for a Ratio of Reaction Rates During the last ten years, Pomraning ${ }^{(26-28)}$ and Stacey $(34-38)$ have developed variational methods for the evaluation of neutron reaction rates, reaction rate ratios, and reactor reactivity worths. The application of these techniques are suitable for critical systems as well as subcritical systems. The advantages of using the variational method rather than that of directly calculating the perturbed flux and computing the parameter of interest are threefold: (35) (a) the numerical computations for forward and adjoint trial functions. (reference fluxes) might be more accurate than that for the perturbed system, (b) the variational functional is itself a stationary property exploiting method; it provides an accuracy of second order in the difference of the exact (perturbed) and approximate (reference) functions, (c) the use of trial functions in the variational functional provides an economical means for the estimation of the parameter of interest at a given perturbed state. Equation (1.1) is the ratio of two reaction rates; thusly, by the conventional variational method, the variational functional is: $(27,37)$

$$
E_{\gamma}\left[\bar{\phi}, \bar{\theta}^{\star}\right]=\frac{\left\langle\Sigma_{d} \bar{\phi}^{\prime}\right.}{\left\langle\Sigma_{f^{\prime}} \bar{\phi}^{\prime}\right.}+\left\langle\bar{\theta}^{\star}, S-H \bar{\phi}\right\rangle,
$$

where $\bar{\theta}^{\star}$ is mathematically called a Lagrangian multiplier; ${ }^{(40)}$ and in neutron physics, it is an importance function. ${ }^{(20)}$ It is shown (see Appendix A) that Equation (1.4) is a variational principle for $W_{n}[\phi]$ and the stationary conditions for Equation (1.4) are: 


$$
\begin{gathered}
4 \\
H_{\phi}=S, \\
H^{*} \theta^{*}=G^{-}(\phi), \\
G^{-}(\phi)=\frac{\Sigma_{d^{<f_{f}}}<>-\Sigma_{f^{<d}} \Sigma^{\phi>}}{\left\langle\Sigma_{f^{\phi>}}{ }^{2}\right.},
\end{gathered}
$$

where $H^{\star}$ is the adjoint Boltzmann transport operator. ${ }^{(4)}$

The second term of Equation (1.4) is called the correction term. It corrects the difference ${ }^{(35)}$ between the perturbed (exact) flux and the reference (approximate) flux.

To show that Equation (1.4) is of second order in difference of $f 1$ uxes, we substitute

$$
\begin{gathered}
\bar{\phi}=\phi-\delta \phi, \\
\bar{\theta}^{\star}=\theta^{\star}-\delta \theta^{\star},
\end{gathered}
$$

where $\bar{\phi}$ and $\bar{\theta}^{*}$ are trial (reference) forward and adjoint flux respectively, into Equation (1.4), then:

$$
\begin{aligned}
& E_{1}\left[\bar{\phi}_{1} \bar{\theta}^{\star}\right]=\frac{\left\langle\Sigma_{d^{\phi}}\right\rangle-\left\langle\Sigma_{d \phi\rangle}\right.}{\left\langle\Sigma_{f} \phi\right\rangle-\left\langle\Sigma_{f} \delta \phi\right\rangle}+\left\langle\theta^{*}-\delta \theta^{*}, S-H(\phi-\delta \theta)\right\rangle \\
& \left.=\frac{\left\langle\Sigma_{d} \phi\right\rangle}{\left\langle\Sigma_{f} \phi\right\rangle} \cdot \frac{1-\frac{\left\langle\Sigma_{d} \delta \phi\right.}{\left\langle\Sigma_{d \phi\rangle}\right.}}{1-\frac{\left\langle\Sigma_{f} \delta \phi\right\rangle}{\left\langle\Sigma_{f} \phi\right\rangle}}\right]+\left\langle\dot{\theta}^{\star}, S\right\rangle \\
& -\left\langle\delta \theta^{\star}, S\right\rangle-\left\langle\theta^{*}, H \phi\right\rangle+\left\langle\delta \theta^{*}, H_{\phi}\right\rangle \\
& +\left\langle\theta^{*}, H \delta \phi\right\rangle-\left\langle\delta \theta^{*}, H \delta \phi\right\rangle .
\end{aligned}
$$


Since $\left\langle\Sigma_{f} \delta \phi\right\rangle$ is first order in approximation, ${ }^{(13)}$ the terms higher than second order expanded in the bracket could be neglected, hence

$$
\begin{aligned}
& E_{1}\left[\bar{\phi}, \bar{\theta}^{*}\right]=\frac{\left\langle\Sigma_{d} \phi\right\rangle}{\left\langle\Sigma_{f} \phi\right\rangle}-\left\langle\frac{\Sigma_{d^{2}}\left\langle\Sigma_{f} \phi\right\rangle-\Sigma_{f}\left\langle\Sigma_{d} \phi\right\rangle}{\left\langle\Sigma_{f} \phi\right\rangle}{ }^{2}, \delta \phi\right\rangle \\
& +\left\langle\theta^{*}, \mathrm{~S}\right\rangle-\left\langle\delta \theta^{\star}, \mathrm{S}\right\rangle-\left\langle\theta^{\star}, \mathrm{S}\right\rangle+\left\langle\delta \theta^{\star}, \mathrm{S}\right\rangle+ \\
& \left\langle H^{\star} \theta^{\star}, \delta \phi\right\rangle-\left\langle\delta \theta^{\star}, H \delta \phi\right\rangle, \\
& E_{1}\left[\bar{\phi}, \bar{\theta}^{\star}\right]=\frac{\left\langle\Sigma_{d^{\phi}}\right\rangle}{\left\langle\Sigma_{f^{\phi}}\right\rangle}-\left\langle\delta \theta^{\star}, H \delta \phi\right\rangle,
\end{aligned}
$$

where Equations $(1.5),(1.6)$, and the inner product relation:

$$
\left\langle\theta^{\star}, H \phi\right\rangle=\left\langle\phi, H^{*} \theta^{\star}\right\rangle
$$

have been employed. Equation (1.9) shows that first order error in the forward and adjoint fluxes results in second order error of the parameter of interest.

Conventionally, the value of $E_{j}\left[\bar{\phi}, \bar{\theta}^{*}\right]$ is evaluated by solving the forward and adjoint fluxes by Equations (1.10) and (1.11), respectively, for a reference state. Then we use these values in Equation (1.4). The following two cases show the kinds of difficulties that we will meet in the calculations of the fluxes $\bar{\phi}$ and $\bar{\theta}^{*}$ by Equations (1.10) and (1.11)

$$
\bar{H}_{\bar{\phi}}=\bar{S},
$$




$$
\begin{gathered}
\bar{H}^{\star} \bar{\theta}^{\star}=G^{-}(\bar{\phi}), \\
G-(\bar{\phi})=\frac{\bar{\Sigma}_{d}<\bar{\Sigma}_{f} \bar{\phi}>-\bar{\Sigma}_{f}<\bar{\Sigma}_{d} \bar{\phi}>}{<\bar{\Sigma}_{f} \bar{\phi}{ }^{2}}
\end{gathered}
$$

First, the solutions of Equations (1.10) and (1.11) for the variational calculation of the neutron detection efficiency, computed by Equation (1.4), have the problem of the presence of a negative adjoint source in Equation (1.11). It is obvious from the right hand side of Equation (1.11) that at the location of the neutron detector, the value of $G^{-}(\bar{\phi})$ is positive $\left(\bar{\Sigma}_{f}\right.$ is very small for fission chamber), and that in another portion of the reactor, $G^{-}(\bar{\phi})$ is negative ( $\bar{\Sigma}_{d}$ is zero outside the detector position). Second, the source term of Equation (1.11) consists of both the forward and adjoint fluxes $\bar{\phi}$ and $\bar{\theta}^{\star}$. If the estimation of a parameter of interest, like the problem stated in section $A$ of this chapter is done by the variational interpolational method ${ }^{(7)}$ with two reference states, one for forward flux $\bar{\phi}$, the other for adjoint flux $\bar{\theta}^{\star}$, the solution of Equation (1.11) for $\bar{\theta}^{\star}$ needs the value of the forward flux $\bar{\phi}$ at the reference state of the adjoint flux. 


\section{Objectives}

There are three objectives in this dissertation.

The first objective is to develop a variational functional, * for the evaluation of the neutron detection efficiency by the variational technique. The Lewins' type variational functional is a ratio of two other variational functions, one for the numerator of Equation (1.1), i.e.; $\left\langle\Sigma_{d} \phi\right\rangle$, and the other for the denominator of the same equation, $\left\langle\Sigma_{f} \phi\right\rangle$. The proposed technique needs minimum of one forward flux and two adjoint fluxes.

The second objective is to show the calculational procedures which are required in the determination of a parameter evaluated by the proposed technique.

The third objective of this dissertation is the demonstration of the high flexibility of the variational functional form by using the ideas of variational interpolational method, (7) variational extrapolational method, and multi-reference-state variational method, (8) and the conventional variational method, to solve the problem described in section $A$ of this chapter at various perturbation states.

*. Lewius first proposed a similar variational principle ${ }^{(21)}$ for ratios in critical systems. 


\section{CHAPTER II}

\section{MATHEMATICAL FORMULATION}

A. Introductịon

In this chapter, there are two primary objectives. The first is the formulation of the Lewins' variational functional for the problem of interest. The physical meaning of each correction term in this functional is described, and the equivalence between the Lewins' variational functional and Pomraning's functional is proved. The second objective is to extend the Lewins' functional to the variational interpolation, the variational extrapolation, and the method of multi-reference-state variational method.

\section{B. Lewins' Variational Functional.}

First, we repeat Pomraning's functional Equation (1.4), and the stationary conditions, Equations (1.5) and (1.6) here for later comparison with Lewins' variational functional. The Pomraning's functional is

$$
E_{1}\left[\bar{\phi}, \bar{\theta}^{\star}\right]=\frac{\left\langle\Sigma_{d} \bar{\phi}\right\rangle}{\left\langle\Sigma_{f} \bar{\phi}\right\rangle}+\left\langle\bar{\theta}^{\star}, S-H \bar{\phi}\right\rangle,
$$

and the stationary conditions are

$$
\begin{gathered}
H_{\phi}=S, \\
H^{*} \theta^{\star}=G^{-}(\phi),
\end{gathered}
$$


where

$$
G^{\prime}(\phi)=\frac{\Sigma_{d}\left\langle\Sigma_{f} \phi\right\rangle-\Sigma_{f}\left\langle\Sigma_{d} \phi\right\rangle}{\left\langle\Sigma_{f}\right\rangle^{2}},
$$

and

$H=$ the Boltzmann transport operator for the stationary state,

$\phi=$ the steady state angular neutron flux at energy $E$ and the position $\bar{r}$,

$H^{*}=$ the adjoint Boltzmann transport operator of $H$, $\phi^{*}=$ the steady state angular adjoint flux at energy $E$ and position $\bar{r}$,

$S=$ the external neutron source for the stationary state, and $G^{-}(\phi)=$ the adjoint source for Equation (2.3).

J. Lewins $(21)$ introduced a variational functional for use in estimating ratios of integral quantities in critical systems. Lewins' functional is somewhat different from that functional introduced by Pomraning ${ }^{(28)}$ (Equation (2.1)); however, Dwivedi ${ }^{(9)}$ proved that the two functionals are equivalent for ratios in a critical system. Accordingly, we wish to introduce a lewins' type functional for the problem of interest (Equation $(1.1)$ ). To this end, we consider the numerator and denominator of Equation (1.1) separately. For the numerator, the functional is

$$
F_{1}\left[\bar{\phi}, \bar{\theta}{ }_{1}\right]=\left\langle\Sigma_{d} \bar{\phi}\right\rangle+\left\langle\bar{\theta}{ }_{1}, S-H \bar{\phi}\right\rangle,
$$

and for the denominator

$$
F_{2}\left[\bar{\phi}, \bar{\theta}_{2}\right]=\left\langle\Sigma_{f} \bar{\phi}\right\rangle+\left\langle\bar{\theta}{ }_{2}, S-H \vec{\phi}\right\rangle,
$$


with stationary conditions (see Appendix A for methodology):

$$
\begin{gathered}
H_{\phi}=S, \\
H^{*}{ }^{*}{ }_{1}=\Sigma_{d},
\end{gathered}
$$

for Equation (2.4), and

$$
\begin{gathered}
H_{\phi}=S, \\
H^{*}{ }_{2}^{*}=\Sigma_{f},
\end{gathered}
$$

for Equation (2.5).

The trial flux functions $\bar{\phi}, \bar{\theta}^{\star}{ }_{7}$, and $\bar{\theta}^{\star}{ }_{2}$, which are different from the exact (or the stationary) values of the fluxes $\phi, \theta^{*}{ }_{1}$, and $\theta_{2}^{*}$ solved by Equations (2.6) to (2.9); for Equations (2.4) and (2.5) are solutions of the following equations for reference state (or states,

$$
\begin{gathered}
H_{\bar{\phi}}=\bar{S}, \\
\bar{H} \bar{\theta}^{\star}{ }_{1}=\bar{\Sigma}_{d}, \\
\bar{H} \bar{\theta}^{\star}{ }_{2}=\bar{\Sigma}_{f} .
\end{gathered}
$$

The ratio of the functionals $F_{1}\left[\bar{\phi}, \bar{\theta}^{\star}{ }_{1}\right]$ and $F_{2}\left[\bar{\phi}, \bar{\theta}^{\star}{ }_{2}\right]$ is the Lewins' type variational functional for a ratio of two reaction rates:

$$
E_{2}[\bar{\phi}]=\frac{\left\langle\Sigma_{d} \bar{\phi}\right\rangle+\left\langle\bar{\theta}^{*}{ }_{1}, S-H \bar{\phi}\right\rangle}{\left\langle\Sigma_{f} \bar{\phi}\right\rangle+\left\langle\bar{\theta}^{\star}{ }_{2}, S-H_{\phi}\right\rangle} .
$$


It is clear that, in Equation (2.13), as the trial function $\bar{\phi}$ : becomes the exact function, i.e., with the perturbed flux, the Lewins' functional becomes the exact value of the neutron detection efficiency

$$
E_{2}[\dot{\phi}] \rightarrow \frac{\left\langle\Sigma_{d} \phi>\right.}{\left\langle\Sigma_{f} \phi>\right.}=W_{n}[\phi] .
$$

The physical meaning of the second terms in the numerator and denominator of Equation (2.13) can be made clear by the following relations.

First, we substitute the equation

$$
\phi=\bar{\phi}+\delta \phi .
$$

into Equation (2.6) to obtain

$$
H \delta \phi=S-H \bar{\phi} .
$$

Taking the inner product of Equation (2.14) with $\theta^{*}{ }_{i}(i=1,2)$ and subtracting the inner product of Equations (2.7) and (2.9) with $\delta \phi$ leads to

$$
\begin{gathered}
\left\langle\theta{ }_{i}, S-H \bar{\phi}\right\rangle=\left\langle\Sigma_{i}, \delta \phi\right\rangle \\
\Sigma_{i}=\Sigma_{d}, \Sigma_{f} .
\end{gathered}
$$

Equation (2.15) shows that the second terms in Equation (2.13) are the corrections for the flux change from the reference state. Thus the term $\left\langle\bar{\theta}{ }_{1}, S-H \bar{\phi}\right\rangle$ represents the correction for the neutron count rate in the detector due to the flux difference between the 
perturbed state and the reference state. Similarly, $\left\langle\bar{\theta}{ }_{2}, S-H \bar{\phi}\right\rangle$ represents the correction for the total fission rate in the reactor core.

C. The Equivalence Between the Lewins' Functional and the Pomraning's Functional

In order to mathematically assure the reliability and the applicability of the Lewins' functional Equation (2.13) for the calculation of the ratio of interest, the equivalence between Equation (2.1) and Equation (2.13) is shown below.

We assume that the flux difference between the reference and exact state is first order, then we have the relationship of Equation (2.14),

$$
H \delta \phi=S-H \bar{\phi} .
$$

Before carrying out the proof for the equivalence, one should make sure of the order of the term $\left\langle\bar{\theta} *{ }_{2}, S-H \bar{\phi}\right\rangle$ in Equation (2.13) in terms of the flux difference $\delta \phi$. If the correction term is first order in $\delta \phi$, then the binomal expansion for the denominator of Equation (2.13) divided by $\left\langle\Sigma_{f} \bar{\phi}\right\rangle$ could be approximated by a first order expansion. To show it, we take the inner product of Equation (2.14) with $\bar{\theta} * 2$, then

$$
\left\langle\bar{\theta}{ }_{2}, H \delta \phi\right\rangle=\left\langle\delta \phi, H^{*} \bar{\theta}{ }_{2}\right\rangle=\left\langle\bar{\theta}{ }_{2}, S-H \bar{\phi}\right\rangle ;
$$

hence

$$
\left\langle\bar{\theta}{ }_{2}, S-H \bar{\phi}\right\rangle=\left\langle\delta \phi, H^{\star} \bar{\theta}^{\star}{ }_{2}\right\rangle \cdot
$$


Equation (2.16) shows that the correction term of the denominator in Equation (2.13) is of first order in terms of the flux difference $\delta \phi$. Writing Equation (2.13) as

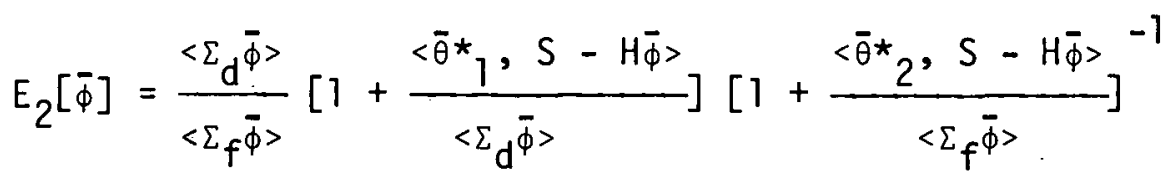

and making use of Equation (2.16), the second bracket on the right hand side of the above equation could be approximated by a first order expansion,

$$
E_{2}[\bar{\phi}]=\frac{\left\langle\Sigma_{d} \bar{\phi}\right\rangle}{\left\langle\Sigma_{f} \bar{\phi}\right\rangle}\left[1+\frac{\left\langle\bar{\theta}^{\star}{ }_{1}, S-H \bar{\phi}\right\rangle}{\left\langle\Sigma_{d} \bar{\phi}\right\rangle}\right]\left[1-\frac{\left\langle\bar{\theta}{ }_{2}, S-H \bar{\phi}\right\rangle}{\left\langle\Sigma_{f} \bar{\phi}\right\rangle}\right] .
$$

From here we expand and collect terms for the right hand side of the above equation to obtain

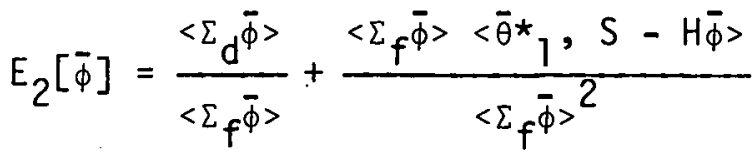

$$
\begin{aligned}
& -\frac{\left\langle\Sigma_{d} \phi\right\rangle\left\langle\bar{\theta}{ }_{2}, S-H \bar{\phi}\right\rangle}{\left\langle\Sigma_{f} \bar{\phi}^{2}\right.} \\
& =W_{n}[\bar{\phi}]+\left\langle\frac{\left\langle\Sigma_{f} \bar{\phi}\right\rangle \bar{\theta}^{*}{ }_{1}-\left\langle\Sigma_{d^{\phi}}\right\rangle^{-*}{ }_{2}}{\left\langle\Sigma_{f} \bar{\phi}\right\rangle^{2}}, S-H \bar{\phi}>.\right.
\end{aligned}
$$

Now we define a new quantity

$$
\bar{\theta} *=\frac{\left\langle\Sigma_{f} \bar{\phi}\right\rangle \bar{\theta}_{1}-\left\langle\sum_{d} \bar{\phi}\right\rangle \bar{\theta}^{*} 2}{\left\langle\left\langle\Sigma_{f} \bar{\phi}\right\rangle^{2}\right.},
$$


then

$$
\mathrm{E}_{2}[\bar{\phi}]=W_{n}[\bar{\phi}]+\langle\bar{\theta} *, S-H \bar{\phi}\rangle .
$$

In Equation (2.18); $\bar{\theta}^{\star}$ is an equivalent adjoint flux or the Lagrangian multiplier for the variational functional $E_{2}[\bar{\phi}] . \bar{\theta}^{*}$. is a value from a reference state. By Taylor's series expansion, we show (see Appendix $B$ ) that $\bar{\theta}^{\star}$ is a first order approximation to the exact value $\theta^{*}$, which is"

$$
\theta^{*}=\frac{\left\langle\Sigma_{f} \phi\right\rangle \theta^{*}{ }_{1}-\left\langle\Sigma_{d^{\phi}} \phi \theta^{*}\right.}{2} .
$$

We are now at a position to show that Equation (2.19) satisfies Equation (2.3), and thus the equality' of Equation (2.18) 'with Equation (2.1). To do this, we operate on Equation (2.19) with $H^{*}$ and make use of Equations (2.7) and (2.9), then

$$
\begin{aligned}
& H^{*} \theta^{*}=\frac{\left\langle\Sigma_{f^{\phi}}>H^{*} \theta_{1}-\left\langle\Sigma_{d^{\phi>}} H^{*} \theta^{*}{ }_{2}\right.\right.}{\left.<\Sigma_{f}\right\rangle^{2}} \\
& =\frac{\Sigma_{d}\left\langle\Sigma_{f} \phi\right\rangle-\Sigma_{f}\left\langle\Sigma_{d} \phi\right\rangle}{\left\langle\Sigma_{f} \phi\right\rangle^{2}},
\end{aligned}
$$

and

$$
H^{*} \theta^{*}=G^{-}(\phi) .
$$

This completes the proof that Equation (2.13) is equivalent to Equation (2.1).

The equivalence of $E_{1}$ and $E_{2}$ can also be shown from the calculational point of view by the superposition principles $(4,17)$ for the solutions of Equation (1.11) (see Appendix C). 


\section{Extensions of the Lewins' Functional}

The advantages of the Lewins' functional are pointed out by comparing Equation (2.13) with Equation (2.1) and their associated equations, i.e., Equations (1.10) and (1.11) as well as Equations (2.10) through (2.12). The first advantage of the Lewins' functional is its flexibility in the choice of reference flux functions. For example, in the conventional variational method, we may choose both the forward and adjoint fluxes at the same reference state; in the variational interpolational method, $(7,39)$ the perturbed state is inside the forward and adjoint reference states; in the variational extrapolational method, the perturbed state is outside the reference states; in the multi-reference-state variational method, we may employ the trial flux functions (forward and adjoint) by linear combination $(8,16,27,29)$ of given reference fluxes. The second advantage is that the solution for the adjoint flux functions is independent of the forward flux function. This is clear when we compare Equations (2.11) and (2.12) with Equation (1.11). In Equations (2.11) and (2.12), there is no dependence on the forward flux, while in Equation (1.11), the source term contains the parameters of $\left\langle\bar{\Sigma}_{d} \bar{\phi}\right\rangle$ and $\left\langle\bar{\Sigma}_{f} \bar{\phi}\right\rangle$. This means that the solution for Equation (1.11) needs the solution of the forward flux $\bar{\phi}$ (or an estimated source) at the same reference state.

In the remainder of this section, the conventional variational method, the variational interpolational method, the variational extrapolational method and the multi-reference-state variational method w111 be presented. 
Conventional Variational Method

Traditionally, both the forward and the adjoint fluxes are chosen at the same reference state. ${ }^{(37)}$ In this case, the calculation of a ratio for a perturbed state which is located on either the left side or the right side of the reference state, is shown in Figure $l(a)$.

The Lewins' functional* for this case is

$$
E_{2}{ }^{C}[\bar{\phi}]=\frac{\left\langle\Sigma_{d} \bar{\phi}_{1}\right\rangle+\left\langle\bar{\theta}{ }_{11}, S-H \bar{\phi}_{1}\right\rangle}{\left\langle\Sigma_{f} \bar{\phi}_{1}\right\rangle+\left\langle\bar{\theta}{ }_{21}, S-H \bar{\phi}_{1}\right\rangle},
$$

where $\bar{\phi}_{1}, \bar{\theta}^{\star}{ }_{11}$, and $\bar{\theta}_{21}$ are obtained respectively from

$$
\begin{aligned}
\bar{H}_{1} \bar{\phi}_{1} & =\bar{S}_{1}, \\
H^{*}{ }_{1} \bar{\theta}_{11} & =\bar{\Sigma}_{\mathrm{d} 1},
\end{aligned}
$$

and

$$
H^{*} \theta^{\theta^{*}}{ }_{21}=\bar{\Sigma}_{f 1} .
$$

Variational Interpolational Method

Steinke, ${ }^{(39)}$ and Cheng and Conn ${ }^{(7)}$ described the concept of the variational interpolational method for the calculation of reactivity and reaction rate, respectively for any perturbation state between two reference states. As shown in Figure $1(b)$, the interpolation is done by calculating the reference forward flux at one of the two reference states and the reference adjoint fluxes at the other one.

*The superscript "C" signifies that this $E_{2}$ is for the conventional variational method. 

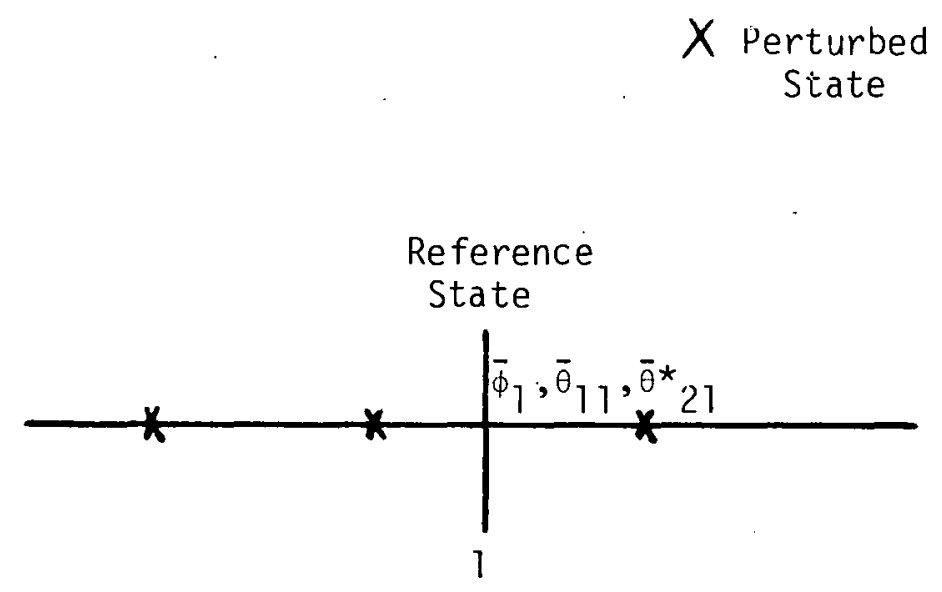

(a) Conventional Variational Method

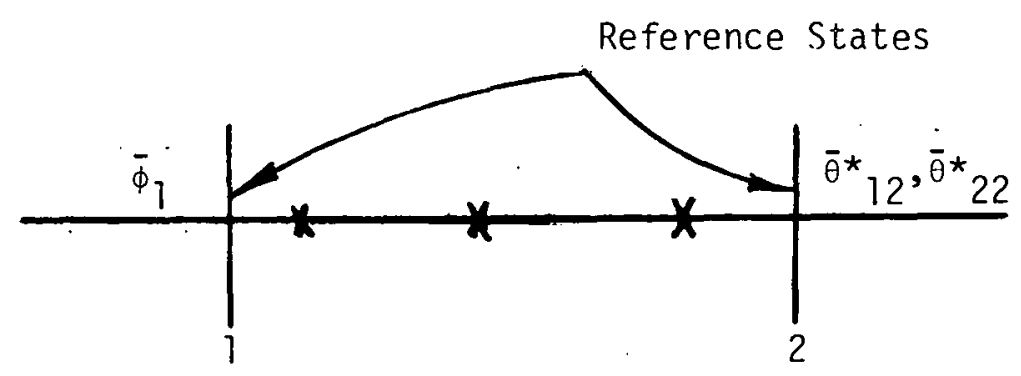

(b) Variational Interpolational Method

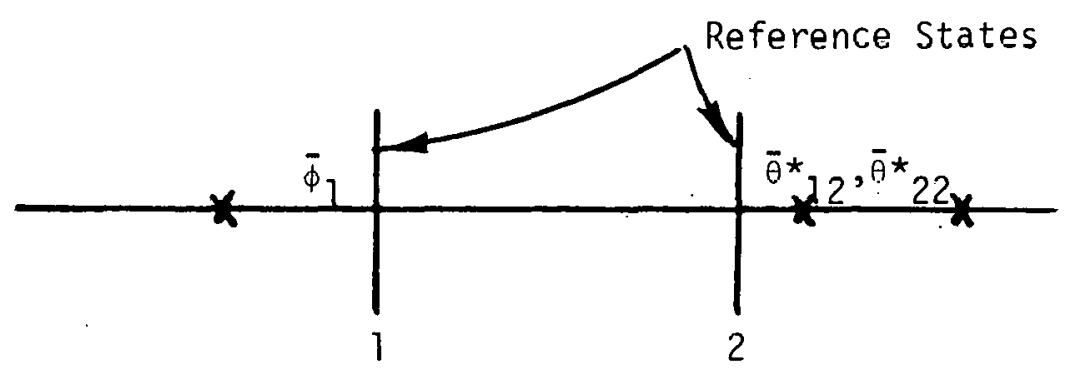

(c) Variational Extrapolational Method

Figure 1. Locations of Reference States and the Perturbed States for Various Variational liethod. 
The Lewins' functional used for this technique is

$$
E_{2}^{I}\left[\bar{\phi}_{1}\right]=\frac{\left\langle\Sigma_{d^{\phi}} \bar{\phi}_{1}+\left\langle\bar{\theta}^{\star}{ }_{12}, S-H \bar{\phi}_{1}\right\rangle\right.}{\left\langle\Sigma_{f} \bar{\phi}_{1}\right\rangle+\left\langle\bar{\theta}_{22}, S-H \bar{\phi}_{1}\right\rangle},
$$

where $\bar{\phi}_{1}, \bar{\theta}^{\star}{ }_{12}$, and $\bar{\theta}_{22}$ are obtained respectively from

$$
\begin{aligned}
\bar{H}_{1} \bar{\phi}_{1} & =\bar{S}_{1}, \\
\bar{H}_{2}{ }_{2}{ }^{\star}{ }_{12} & =\bar{\Sigma}_{\mathrm{d} 2},
\end{aligned}
$$

and

$$
\bar{H} *{ }_{2} \bar{\theta}_{22}=\bar{\Sigma}_{f 2}
$$

Variational Extrapolational Method

This method is the extended concept from the variational interpolational method. The two reference states are located on one side of a perturbed state. Its orientation is shown in Figure $1(c)$ and the functional form utilized here is

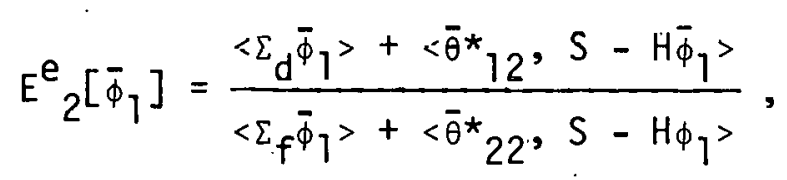

where the reference fluxes are solved by Equations (2.25) through (2.27).

Multi-Reference-State Variational Method

Pomraning ${ }^{(27)}$ and Conn ${ }^{(8)}$ have suggested the applications of the multistate variational method for the calculation of a ratio. According to Conn, ${ }^{(8)}$ the multi-reference-state variational method has a better accuracy even if the values of the reference functions are poor. 
Generally, we choose equal number of reference states for forward and adjoint functions. The locations of reference states may be anywhere in the range of interest, while the perturbed state is within reference states. In the case that the system consists of many reference states, the better trial function for the system might be a linear combination of the known functions. Assume that there are $2 \mathrm{~N}$ reference points, among them, we choose any $\mathrm{N}$ points for forward functions, and the rest for adjoint functions. Thus, the linear combinations of the trial functions for the system are

$$
\phi_{t}(x)=\sum_{i}^{N} a_{i} \bar{\phi}_{j}(x)=\vec{a} \vec{a}_{\phi}^{T}
$$

and

$$
\theta_{t \ell}^{\star}(x)=\sum_{i}^{N} a_{l i}^{\star} \bar{\theta}_{\ell j i}(x)=\vec{a}^{\star}{ }_{l}^{\top} \vec{\theta}_{l},
$$

where

$$
\begin{aligned}
& x= \text { phase space, and } \\
& a_{i}, a^{*}{ }_{i}= \text { combination coefficients of the forward and adjoint trial } \\
& \text { function for the system, respectively. } \\
& \text { If we rewrite Equations }(2.4) \text { and }(2.5) \text { for the system with }
\end{aligned}
$$

combined trial functions:

$$
\begin{aligned}
& F_{1}\left[\phi_{t}, \theta^{\star}{ }_{t 1}\right]=\left\langle\Sigma_{d} \phi_{t}\right\rangle+\left\langle\theta_{t l}^{\star}, S-H_{\phi_{t}}\right\rangle, \\
& F_{2}\left[\phi_{t}, \theta^{*}{ }_{t 2}\right]=\left\langle\Sigma_{f} \phi_{t}\right\rangle+\left\langle\theta_{t 2}^{\star}, s-H_{t}\right\rangle
\end{aligned}
$$

and substitute Equations (2.29) and (2.30) into Equations (2.31) and (2.32), then 


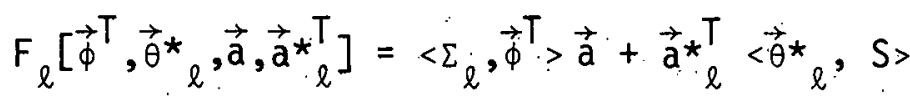

$$
\begin{aligned}
& -\vec{a}^{\top} \star_{i}^{\top<\vec{\theta}^{\star}} e_{\ell}, \vec{H}^{\top}>\vec{a} \\
& \ell=1,2 \text {, } \\
& \Sigma_{\ell}=\Sigma_{d}, \Sigma_{f}:
\end{aligned}
$$

In order to find the stationary values for $\vec{a}$ and $\vec{a}_{\ell}{ }_{l}$, the first variation with respect to $\vec{a}$ and $\vec{a}^{\star}{ }_{\ell}$ are taken which leads to:

$$
<\Sigma_{\ell}, \vec{\phi}^{\top}>-\vec{a}^{\star} \ell^{\top}<\vec{\theta}_{\ell}^{\star}, H^{\top}{ }^{\top}>=0,
$$

or

$$
\vec{a}^{\star}{ }_{\ell}^{\top}=\left\langle\Sigma_{\ell}, \vec{\phi}^{\top}\right\rangle\left\langle\vec{\theta}_{\ell}^{*} ; \vec{H}^{\top}\right\rangle^{-1},
$$

and

$$
\left\langle\vec{\theta}^{\star}, S\right\rangle-\left\langle\vec{\theta}^{\star}{ }_{\ell}, H_{\phi}^{+} \vec{T}^{\dagger}\right\rangle \vec{a}=0,
$$

or

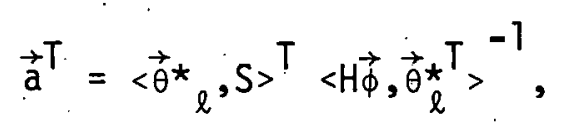

where $<,>^{-1}=$ inverse of a matrix,

and

$$
\begin{aligned}
\ell & =1,2, \\
\Sigma_{\ell} & =\Sigma_{d}, \Sigma_{f} .
\end{aligned}
$$

Substituting Equations (2.34) and (2.36) into Equation (2.33)

we have

$$
F_{\ell}\left[\vec{\phi}, \vec{\theta}_{\ell}^{\star}\right]=\left\langle\vec{\theta} \star_{\ell}^{\top}, S\right\rangle\left\langle H \vec{\phi}, \vec{\theta}_{\ell} \star_{l}^{\top}<\Sigma_{\ell}, \vec{\phi}>\right.
$$


where

$$
\begin{aligned}
\ell & =1,2, \\
\vdots & =\Sigma_{d}, \Sigma_{f},
\end{aligned}
$$

and

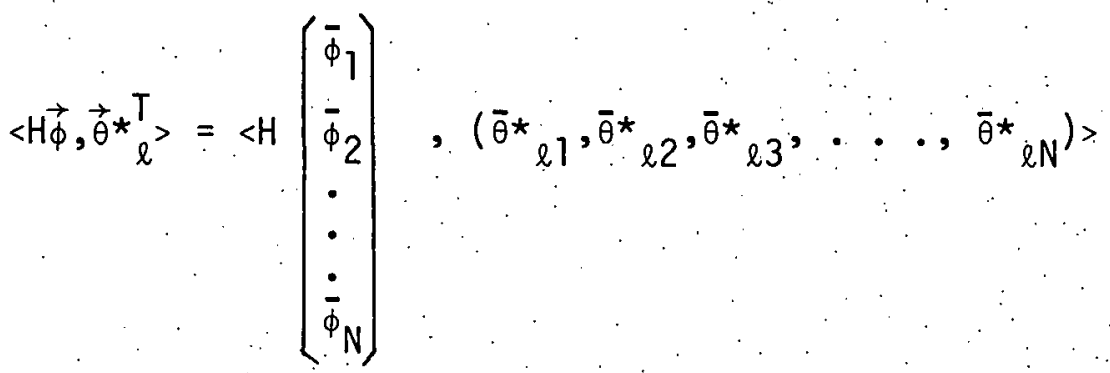

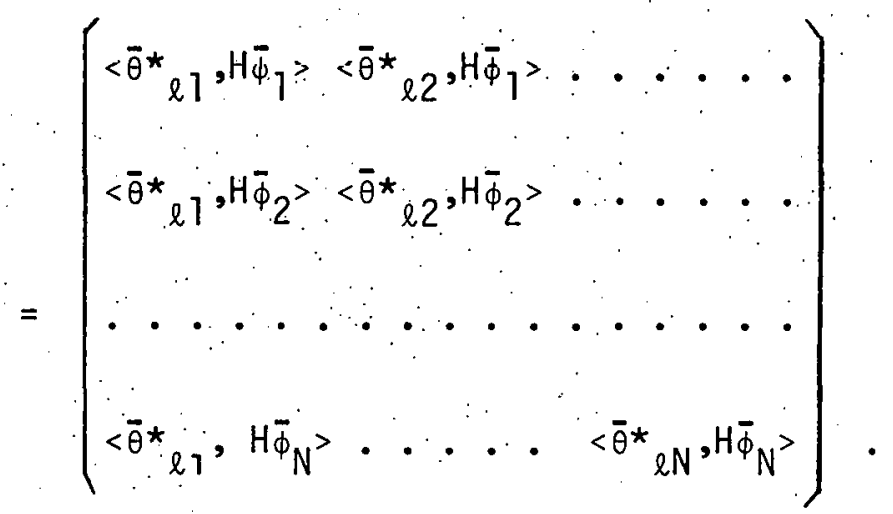

If we substitute Equation (2.37) into Equation (2.13), the Lewins' functional form for the multi-reference-state system becomes

$$
E_{2}^{m}[\vec{\phi}]=\frac{\left\langle\vec{\theta}_{1}^{\top}, S\right\rangle\left\langle H_{\vec{\phi}}, \vec{\theta}^{\top} \star_{1}^{\top}\right\rangle^{-T}\left\langle\Sigma_{d}, \vec{\phi}\right\rangle}{\left\langle\vec{\theta}^{\top}{ }_{2}^{\top}, S\right\rangle\left\langle H_{\vec{\phi}}, \vec{\theta}^{\top} \star_{2}^{\top}\right\rangle^{-1}\left\langle\Sigma_{\vec{f}}, \vec{\phi}\right\rangle}
$$

For calculational purpose, the inner products $\left\langle\bar{\theta}^{\star}{ }_{\ell j}, H_{\phi_{j}}\right\rangle$ may be expressed explicitly by the following relations: 


$$
\begin{aligned}
S-H \bar{\phi} & =S-H \bar{\phi}+(\bar{H} \bar{\phi}-\bar{S}) \\
& =(S-\bar{S})-(H-\bar{H}) \bar{\phi} \\
& =\Delta S-\Delta H \bar{\phi}, \\
\Delta H \bar{\phi} & =\Delta S-S+H \bar{\phi} \\
= & -\bar{S}+H \bar{\phi}, \\
\dot{H} \bar{\phi}= & \bar{S}+\Delta H \bar{\phi},
\end{aligned}
$$

and then

$$
\begin{aligned}
\left\langle\bar{\theta}_{\ell j}^{\star}, H \bar{\phi}_{j}\right\rangle & =\left\langle\bar{\theta}_{\ell j}, \bar{S}+\Delta H_{\phi_{j}}\right\rangle \\
& =\left\langle\bar{\theta}_{\ell j}, \bar{S}\right\rangle+\left\langle\bar{\theta}_{\ell j}, \Delta H \bar{\phi}_{j}\right\rangle .
\end{aligned}
$$


CHAPTER I I

COMPUTATIONAL PROCEDURES

A. Introduction

The objectives of this chapter are to formulate a numerical. form of the Lewins' functional for computational purpose and to describe the calculational procedures that are required in the execution of the variational functional.

\section{B. The Numerical Form of the Lewins' Functional}

For reference purpose, we rewrite Equation (2.13) here

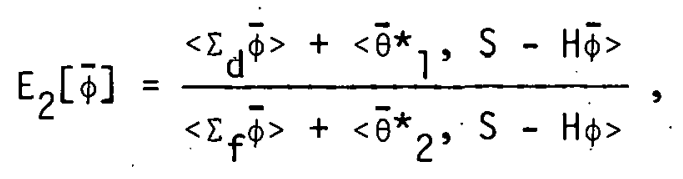

and for convenience, we write the numerator and the denominator of Equation (3.1) as.

$$
F_{i}\left[\bar{\phi}, \bar{\theta}{ }_{i}\right]=\left\langle\Sigma_{i} \bar{\phi}\right\rangle+\left\langle\bar{\theta}{ }_{i}, S-H \bar{\phi}\right\rangle,
$$

where

$$
\begin{aligned}
i & =1,2 \text {, and } \\
\Sigma_{i} & =\Sigma_{d}, \Sigma_{f} .
\end{aligned}
$$

The trial neutron flux functions, $\bar{\phi}, \bar{\theta}^{\star}{ }_{j}$, in Equation (3.2) are respectively the solution of the following equations 


$$
\begin{gathered}
24 \\
H_{\bar{\phi}}=\bar{S},
\end{gathered}
$$

and

$$
H * \bar{\theta}{ }_{i}=\bar{\Sigma}_{i} \cdot
$$

In order to express Equations (3.2) in a detailed numerical form, we write Equations (3.3) and (3.4) explicitly as:

$$
\begin{aligned}
& \bar{\Omega} \cdot \nabla \bar{\phi}(\bar{r}, E, \bar{\Omega})+\bar{\Sigma}(\bar{r}, E) \bar{\phi}(\bar{r}, E, \bar{\Omega})= \\
& \frac{X(E)}{4 \pi} \int \rho \overline{v \Sigma_{f}}\left(\vec{r}, E^{-}\right) \bar{\phi}\left(\bar{r}, E^{-}, \vec{\Omega}^{-}\right) \mathrm{dE}^{-} \mathrm{d} \bar{\Omega}^{-}+ \\
& \iint \bar{\Sigma}_{\mathrm{S}}\left(\bar{r} ; \mathrm{E}^{-}, \bar{\Omega}^{-} \rightarrow \overline{\mathrm{E}}, \bar{\Omega}\right) \bar{\phi}\left(\bar{r}, \mathrm{E}^{-}, \bar{\Omega}^{-}\right) \mathrm{dE}^{-} \mathrm{d} \bar{\Omega}^{-}+\overline{\mathrm{S}}(\bar{r}, \mathrm{E}, \bar{\Omega}),
\end{aligned}
$$

and

$$
\begin{aligned}
& -\bar{\Omega} \cdot \nabla \bar{\theta}{ }_{i}(\bar{r}, E, \bar{\Omega})+\bar{\Sigma}(\bar{r}, E) \bar{\theta}^{\star}{ }_{j}(\bar{r}, E, \bar{\Omega})= \\
& \frac{\overline{\nu \Sigma_{f}}(\bar{r}, E)}{4 \pi} \int S X\left(E^{-}\right) \bar{\theta}^{\star}{ }_{j}\left(\bar{r}, E^{-}, \bar{\Omega}^{-}\right) \mathrm{dE}^{-} \mathrm{d} \bar{\Omega}^{-}+ \\
& \iint \bar{\Sigma}_{\mathrm{S}}\left(\bar{r} ; \mathrm{E}, \bar{\Omega} \rightarrow \mathrm{E}^{-}, \bar{\Omega}^{-}\right) \bar{\theta}^{\star}{ }_{i}\left(\bar{r}, \mathrm{E}^{-}, \bar{\Omega}^{-}\right) \mathrm{dE} \mathrm{E}^{-} \mathrm{d} \bar{\Omega}^{-}+\bar{\Sigma}_{j}(\bar{r}, \mathrm{E}) . .
\end{aligned}
$$

Over the reactor system, on which the parameters of interest are evaluated, the boundary conditions ${ }^{(4,42)}$ for Equations (3.5) and (3.6) are shown in Table 1.

Equation (3.5) and Equation (3.6) are energy dependent Boltzmann neutron transport equations. In practical calculations, the energy variable can be treated by multigroup method, $(4,25)$

*The adjoint source of Equation (3.4) is a macroscopic cross section which is usually independent of neutron direction. 
TABLE 1

BOUNDARY CONDITIONS FOR FORWARD AND ADJOINT

NEUTRON TRANSPORT EQUATIONS

Forward Equation

1. $\bar{\phi}\left(\bar{r}_{\mathrm{b}}, E, \bar{\Omega}\right)^{\mathrm{a}}=0, \quad \overline{\mathrm{n}}_{\mathrm{in}} \cdot \bar{\Omega}>0$.

2. $\bar{\phi}(\bar{r}, E, \bar{\Omega})$ is continuous in the system.

3. $\bar{\phi}(\bar{r}, E, \bar{\Omega})$ is finite in the system.

Adjoint Equation

1. $\bar{\theta} \star_{j}\left(\bar{r}_{b}, E, \bar{\Omega}\right)=0, \bar{n}_{\text {out }} \cdot \bar{\Omega}>0$.

2.. $\bar{\theta}^{\star}{ }_{j}(\bar{r}, E, \bar{\Omega})$ is continuous in system.

3. $\bar{\theta}^{\star}{ }_{i}(\bar{r}, E, \bar{\Omega})$ is finite in the system. system.

a $\bar{r}_{b}$ represents the boundary coordinates of the reactor 
that is, the neutron energy range of interest is divided into a number of subintervals, each subinterval represents an energy group, and each group has its own specified properties and governing equation.

In order to do this, we make an integration for Equations (3.5) and (3.6) over a definite energy interval, then we have multigroup neutron transport equations:

$$
\begin{aligned}
& \bar{\Omega} \cdot \nabla \bar{\phi}_{\mathrm{g}}(\bar{r}, \bar{\Omega})+\bar{\Sigma}_{\mathrm{g}}(\bar{r}) \bar{\phi}_{\mathrm{g}}(\bar{r}, \bar{\Omega})= \\
& \frac{X_{g}}{4 \pi} \cdot g_{g^{-}}^{G}\left(\overline{v \Sigma_{f}}(\bar{r})\right)_{g^{-}} f \bar{\phi}_{g^{-}}\left(\bar{r}, \bar{\Omega}^{-}\right) \mathrm{d} \bar{\Omega}^{-} .
\end{aligned}
$$

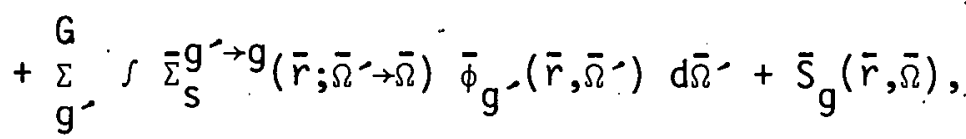

for Equation (3.5), and

$$
\begin{aligned}
& -\bar{\Omega} \cdot \bar{\theta}^{*}{ }_{i g}(\bar{r}, \bar{\Omega})+\bar{\Sigma}_{g}(\bar{r}) \bar{\theta}^{\star}{ }_{i g}(\bar{r}, \bar{\Omega})=
\end{aligned}
$$

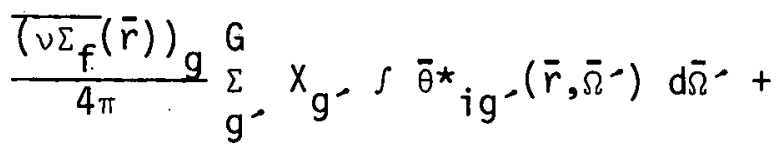

$$
\begin{aligned}
& \sum_{g^{\prime}}^{G} \int \bar{\Sigma}_{S}^{g^{\rightarrow} g^{-}}\left(\bar{r} ; \bar{\Omega}+\bar{\Omega}^{-}\right) \bar{\theta}^{*}{ }_{i g^{\prime}}\left(\bar{r}, \bar{\Omega}^{\prime}\right) \mathrm{d} \bar{\Omega}^{-}+\bar{\Sigma}_{i g}(\bar{r}),
\end{aligned}
$$

for Equation (3.6),

where

$$
\begin{gathered}
\bar{\phi}_{g}(\bar{r}, \bar{\Omega})=\int_{E_{g}}^{E g-1} \bar{\phi}(\bar{r}, E, \bar{\Omega}) d E, \\
\bar{\theta}^{\star}{ }_{i g}(\bar{r}, \bar{\Omega})=\int_{E_{g} g-1}^{E} \bar{\theta}_{i}(\bar{r}, E, \bar{\Omega}) d E,
\end{gathered}
$$




$$
\begin{aligned}
& \bar{\Sigma}_{g}(\bar{r})=\int_{E_{g}}^{E} g-1 \bar{\Sigma}(\bar{r}, E) \bar{\phi}(\bar{r}, E, \bar{\Omega}) \mathrm{dE} / \bar{\phi}_{g}(\bar{r}, \bar{\Omega}), \\
& \left.\left.\overline{\left(\nu \Sigma_{f}\right.}(\bar{r})\right)_{g}=\int_{E_{g}}^{E} g-1\right) \overline{\left(\nu \Sigma_{f}\right.}(\bar{r}, E) \bar{\phi}(\bar{r}, E, \bar{\Omega}) \mathrm{dE} / \bar{\phi} \cdot(\bar{r}, \bar{\Omega}), \\
& x_{g}=\int_{E}^{E} g-1 \times(E) d E \\
& \bar{\Sigma}_{S}^{g^{\circ} \rightarrow g}\left(\bar{r} ; \bar{\Omega}^{-} \rightarrow \bar{\Omega}\right)=\int_{E_{g}}^{E} g-1 \quad \int_{E_{g^{\prime}}}^{E^{\prime}-1} \bar{\Sigma}_{S}\left(\bar{r} ; E^{\prime}, \bar{\Omega}^{-} \rightarrow E, \bar{\Omega}\right) . \\
& \bar{\phi}\left(\bar{r}, E^{-}, \bar{\Omega}^{-}\right) d E^{-} d E / \bar{\phi} g^{-}\left(\bar{r}, \bar{\Omega}^{-}\right), \\
& \bar{S}_{g}(\bar{r}, \bar{\Omega})=\int_{E_{g}}^{E} g-1 \bar{S}(\bar{r}, E, \bar{\Omega}) \mathrm{dE},
\end{aligned}
$$

and

$$
\bar{\Sigma}_{i g}(\bar{r})=\int_{E_{g}}^{E} g-1 \bar{\Sigma}_{j}(\bar{r}, E) d E .
$$

Practically, the parameters for the multigroup adjoint-flux equation are obtained by.constructing the adjoint operator for the multigroup adjoint equation directly from the multigroup forward equations.

In order to cancel the convective term of the Boltzmann equation, we add $(\bar{H} \bar{\phi}-\bar{S})$ to the second term of Equation (3.2), and after subtracting, we have

$$
F_{i}\left[\bar{\phi}, \bar{\theta}^{*}{ }_{i}\right]=\left\langle\Sigma_{i} \bar{\psi}\right\rangle+\left\langle\bar{\theta}^{\star}{ }_{j}, \Delta S-\Delta H \bar{\phi}\right\rangle,
$$

where

$$
\begin{aligned}
\Delta S & =S-\bar{S}, \\
\Delta H & =H-A, \text { the change of the transport operator. }
\end{aligned}
$$


The change of a state is due to the changes of material parameters of the system, from Equation (3.5), we have

$$
\begin{aligned}
& \Delta H(\bar{r}, E, \bar{\Omega}) \bar{\phi}(\bar{r}, E, \bar{\Omega})=\Delta \Sigma(\bar{r}, E) \bar{\phi}(\bar{r}, E, \bar{\Omega})- \\
& \iint \Delta \Sigma_{s}\left(\bar{r} ; E^{-}, \bar{\Omega}^{-} \rightarrow E, \bar{\Omega}\right) \bar{\phi}\left(\bar{r}, E^{-}, \bar{\Omega}^{-}\right) \mathrm{dE} E^{-} \mathrm{d} \bar{\Omega}^{-} \\
& -\frac{X(E)}{4 \pi} \int S \Delta\left(\nu \Sigma_{f}\left(\bar{r}, E^{-}\right)\right) \bar{\phi}\left(\bar{r}, E^{-}, \bar{\Omega}^{-}\right) \mathrm{d} E^{-} \mathrm{d} \bar{\Omega}^{-},
\end{aligned}
$$

when Equation (3.20) is substituted into Equation (3.17) and summed over energy groups, the result is an angular and spatial dependent functional

$$
\begin{aligned}
& F_{i}\left[\bar{\phi}_{g}, \bar{\theta}^{\star}{ }_{i g}\right]=\sum_{g=1}^{G} \iint \bar{\phi}_{g}(\bar{r}, \bar{\Omega}) \Sigma_{i g}(\bar{r}) d \bar{r} d \bar{\Omega}+ \\
& \sum_{g=1}^{G} \int S \bar{\theta}^{\star}{ }_{i g}(\bar{r}, \bar{\Omega}) \Delta S_{g}(\bar{r}, \bar{\Omega}) d \bar{r} d \bar{\Omega}- \\
& \sum_{g=1}^{G} \iint \Delta \Sigma_{g}(\bar{r}) \bar{\theta}^{\star}{ }_{i g}(\bar{r}, \bar{\Omega}) \bar{\phi}_{g}(\bar{r}, \bar{\Omega}) \mathrm{d} \bar{r} \mathrm{~d} \bar{\Omega}+
\end{aligned}
$$

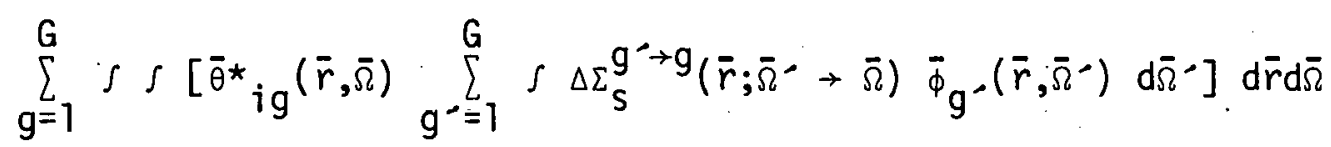

$$
\begin{aligned}
& +\sum_{g=1}^{G} \frac{X_{g}}{4 \pi} s \int\left[\bar{\theta}{ }_{i g}(\bar{r}, \bar{\Omega}) \sum_{g=1}^{G} \Delta\left(\nu \Sigma_{f}(\bar{r})\right)_{g}-\bar{\phi}_{g} \cdot\left(\bar{r}, \bar{\Omega}^{\prime}\right) d \bar{\Omega}^{-}\right] d \bar{r} d \bar{\Omega} .
\end{aligned}
$$


We expand fluxes, scattering cross sections, and sources in spherical harmonic functions as $(4,15,23)$

$$
\begin{aligned}
& \phi_{g}(\bar{r}, \bar{\Omega})=\sum_{n=0}^{\text {ISCT }}\left(\frac{2 n+1}{4 \pi}\right) \sum_{K=0}^{n} \bar{\phi}_{g n}^{-K}(\bar{r}) \gamma_{n}^{K}(\bar{\Omega}), \\
& \bar{\theta}_{i g}^{*}(\bar{r}, \bar{\Omega})=\sum_{\ell=0}^{\operatorname{ISCT}}\left(\frac{2 \ell+1}{4 \pi}\right) \sum_{m=0}^{\ell} \bar{\theta}_{i g \ell} \star_{i g}^{m}(\bar{r}) \gamma_{\ell}^{m}(\bar{\Omega}), \\
& \Delta \Sigma_{S}^{g^{\prime} \rightarrow g}\left(\bar{r} ; \bar{\Omega}^{-} \rightarrow \bar{\Omega}\right)=\Delta \Sigma_{S}^{g^{\prime} \rightarrow g}\left(\bar{r} ; \bar{\Omega} \cdot \bar{\Omega}^{-}\right) \\
& =\sum_{n=0}^{\operatorname{ISCT}}\left(\frac{2 n+l}{4 \pi}\right) \Delta \Sigma_{S n}^{g^{\prime} \rightarrow g}(\bar{r}) P_{n}\left(\bar{\Omega} \cdot \bar{\Omega}^{-}\right),
\end{aligned}
$$

and

$$
\Delta S_{g}(\bar{r}, \bar{\Omega})=\sum_{n=0}^{\text {ISCT }}\left(\frac{2 n+1}{4 \pi}\right) \sum_{K=0}^{n} \Delta S_{g n}^{K}(\bar{r}) \gamma_{n}^{K}(\bar{\Omega}),
$$

respectively, where the normalized spherical harmonics is defined by

$$
\begin{aligned}
& Y_{n}^{K}(\vec{\Omega})=\left[\frac{\left(2-\delta_{n 0}\right)(n-K) !}{(n+K) !}\right]^{1 / 2} P_{n}^{K}(\mu) \cos K \Phi, \\
\delta_{K .0}= & 1, \text { if } K=0, \\
= & 0, \text { if } K \neq 0,
\end{aligned}
$$

and make use of the addition theorem (22)

$$
P_{n}(\bar{s} \cdot \bar{s} \cdot \cdot)=\sum_{K=0}^{n} Y_{n}^{K}(\bar{\Omega}) Y_{n}^{K}(\bar{\Omega} \cdot) .
$$


Substitute Equations (3.22-3.25) and (3.27) into Equation (3.21) and make use of the relationship

$$
\delta_{4 \pi} Y_{n}^{K}(\bar{\Omega}) Y_{\ell}^{m}(\bar{\Omega}) \mathrm{d} \bar{\Omega}=\frac{4 \pi}{2 n+1} \delta_{n \ell} \delta_{K m},
$$

to obtain

$$
\begin{aligned}
& F_{i}\left(\bar{\phi}_{g}, \bar{\theta}^{*}{ }_{i g}\right]=\sum_{g=1}^{G} \sum_{i j}^{I J} \bar{\phi}_{g}\left(\bar{r}_{i j}\right) \Sigma_{i g}\left(\bar{r}_{i j}\right) v_{i j}+ \\
& \sum_{g=1}^{G} \sum_{n=0}^{I S C T}\left(\frac{2 n+1}{4 \pi}\right) \sum_{K=0}^{n} \sum_{i j}^{I J} \bar{\theta}_{i g n}^{K}\left(\bar{r}_{i j}\right) \Delta S_{g n}^{K}\left(\bar{r}_{i j}\right)- \\
& \sum_{g=1}^{G} \sum_{n=0}^{I S C T}\left(\frac{2 n+l}{4 \pi}\right) \sum_{K=0}^{n} \sum_{i j}^{I J} \Delta \Sigma g\left(\bar{r}_{i j}\right) \bar{\theta}^{\star K}{ }_{i g n}\left(\bar{r}_{i j}\right) \bar{\phi}_{g n}^{K}\left(\bar{r}_{i j}\right) v_{i j}+ \\
& \sum_{n=0}^{I S C T}\left\{\left(\frac{2 n+1}{4 \pi}\right) \sum_{i j}^{I J} V_{i j}\left[\sum_{g=1}^{G} \sum_{K=0}^{n} \bar{\theta}^{\star K}{ }_{i g n}\left(\bar{r}_{i j}\right) \sum_{g^{-}=1}^{G} \Delta \Sigma_{s n}^{g} \rightarrow g\left(\bar{r}_{i j}\right) \bar{\phi}_{g}^{K} \cdot{ }^{K}\left(\bar{r}_{i j}\right)\right]\right\} \\
& +\sum_{g=1}^{G} \frac{X_{g}}{4 \pi} \sum_{i j}^{I j} V_{i j}\left[\bar{\theta}^{\star}{ }_{i g}\left(\bar{r}_{i j}\right) \sum_{g^{\prime}=1}^{G} \Delta\left(v \Sigma_{f}\left(\bar{r}_{i j}\right)\right)_{g^{\prime}} \cdot \bar{\phi}_{g^{\prime}}\left(\bar{r}_{i j}\right)\right]
\end{aligned}
$$

where

$$
\begin{aligned}
G & =\text { total neutron energy groups, } \\
\text { ISCT } & =\text { the order of scattering, } \\
V_{i j} & =\text { the volume of the } i j^{\text {th }} \text { interval, } \\
I J & =\text { total number of intervals. }
\end{aligned}
$$

The application of the forward and adjoint flux moments in Equation (3.29) is presently used for the two-dimensional problem of sensitivity analysis. ${ }^{(44)}$ The use of flux moments instead of angular 
flux in two-dimensional system has a desirable advantage of avoiding the storage of a large number of neutron angular fluxes. The code DOT offers both the forward and the adjoint flux moments, while the code ANISN offers angular fluxes only. So, for one-dimensional problems, the numerical form (see Appendix D) for the variational functional Equation (3.21) employs neutron angular fluxes.

\section{Calculational Procedures}

The solution of Equation (3.1) is carried out by evaluating both the numerator and denominator separately, as dictated by Equation (3.29). The forward and the adjoint reference fluxes are calculated by solving Equations (3.5) and (3.6), respectively. The numerical calculation for Equation (3.29) has two steps: (a) calculate the first term with the reference forward flux and the nuclear parameters at the perturbed state, (b) evaluate the remainders of the equation (i.e., the correlation term of Equation (3.2)), by using the sensitivity code SWANLAKE ${ }^{(3)}$ and the changes of the nuclear parameters from the reference state.

The calculation of both the forward and the adjoint fluxes at reference states will be done by ANISN ${ }^{(10)}$ for one-dimensional problems, and by DOT ${ }^{(27)}$ for two-dimensional problems. The SWANLAKE code calculates the products of the adjoint and the forward $(3)$ $\left(\phi^{*} \phi\right)$ flux moments for one-dimensional problems by directly inputting the angular fluxes. For two-dimensional problem, an intermediate code VIP ${ }^{(6)}$ is used to calculate the products of the adjoint and the forward flux moments. SWANLAKE uses the moment products as input. 


\section{CHAPTER IV.}

\section{NUMERICAL DEMONSTRATIONS}

\section{A. Introduction}

There are two classes of problems to be demonstrated in this chapter. Each $c l a s s$ has at least two of the four types of calculational methods, i.e., the variational interpolation, the variational extrapolation, the conventional variational, and the multi-reference-state variational method.

The first class are one-dimensional problems. The neutron fluxes (forward and adjoint) are calculated by the $\operatorname{ANISN}(10)$ code with $P_{3} S_{4}$ : The 50 -group neutron cross section set $(45,46)$ prepared for the Engineering Mockup Critical (EMC) facility was employed. The second class consists of two-dimensional problems. The neutron fluxes involved in the variational calculations for this second class were calculated by the discrete ordinate code $00 T^{(31)}$ with $\mathrm{P}_{3} \mathrm{~S}_{4}$. The 14-group cross section set used for the two-dimensional problems were collapsed from the 50-group sets used for EMC by the ANISN code. The correction terms for the neutron flux change from a reference state, i.e., the second terms in Equation (2.13), were evaluated by the sensitivity code SWANLAKE ${ }^{(3)}$ for the two classes of problems. 


\section{B. One-Dimensional Calculations}

\section{Description}

The one-dimensional calculation of the detection efficiency $\left(W_{n}\right)$ in this dissertation used the slab model shown in Figure 2. This model is a mockup of the EMC shown in Figure 3 . The calculation of $D E$ was done for one gram of active material in the detector. Thusly, from Equation (1.1), we have

$$
\begin{aligned}
& W_{n}[\phi]=\frac{\left\langle\Sigma_{d} \phi\right\rangle}{\left\langle\Sigma_{f} \phi\right\rangle}
\end{aligned}
$$

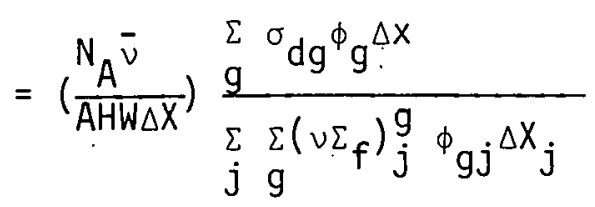

$$
\begin{aligned}
& =\left(\frac{N_{A} \bar{v}}{A H W}\right) \frac{\sum^{\sigma_{d g} \phi_{g}^{\prime}}}{\sum_{j} \sum_{g}\left(\nu \Sigma_{f}\right)_{j}^{g} \phi_{g j} \Delta X_{j}},
\end{aligned}
$$

where

$$
\begin{aligned}
N_{A}= & \text { Avogadro's number, } \\
\bar{v}= & \text { average number of neutrons per fission, } \\
A= & \text { mass number of active material, } \\
H= & \text { height of the slab, } \\
W= & \text { width of the slab, } \\
\sigma_{d g}= & \text { group microscopic cross section in detector, } \\
\left(\nu \Sigma_{f}\right)_{j}^{g}= & \text { product of the number of neutrons per fission and the } \\
& \text { macroscopic fission cross section for the } g^{\text {th }} \text { group and } \\
& \text { the } j \text { th interval. }
\end{aligned}
$$


CR: Control Rod

SR: Safety Rod

GP: General Purpose Loop

MT: Material Test Loop

SP: Special Purpose Loop

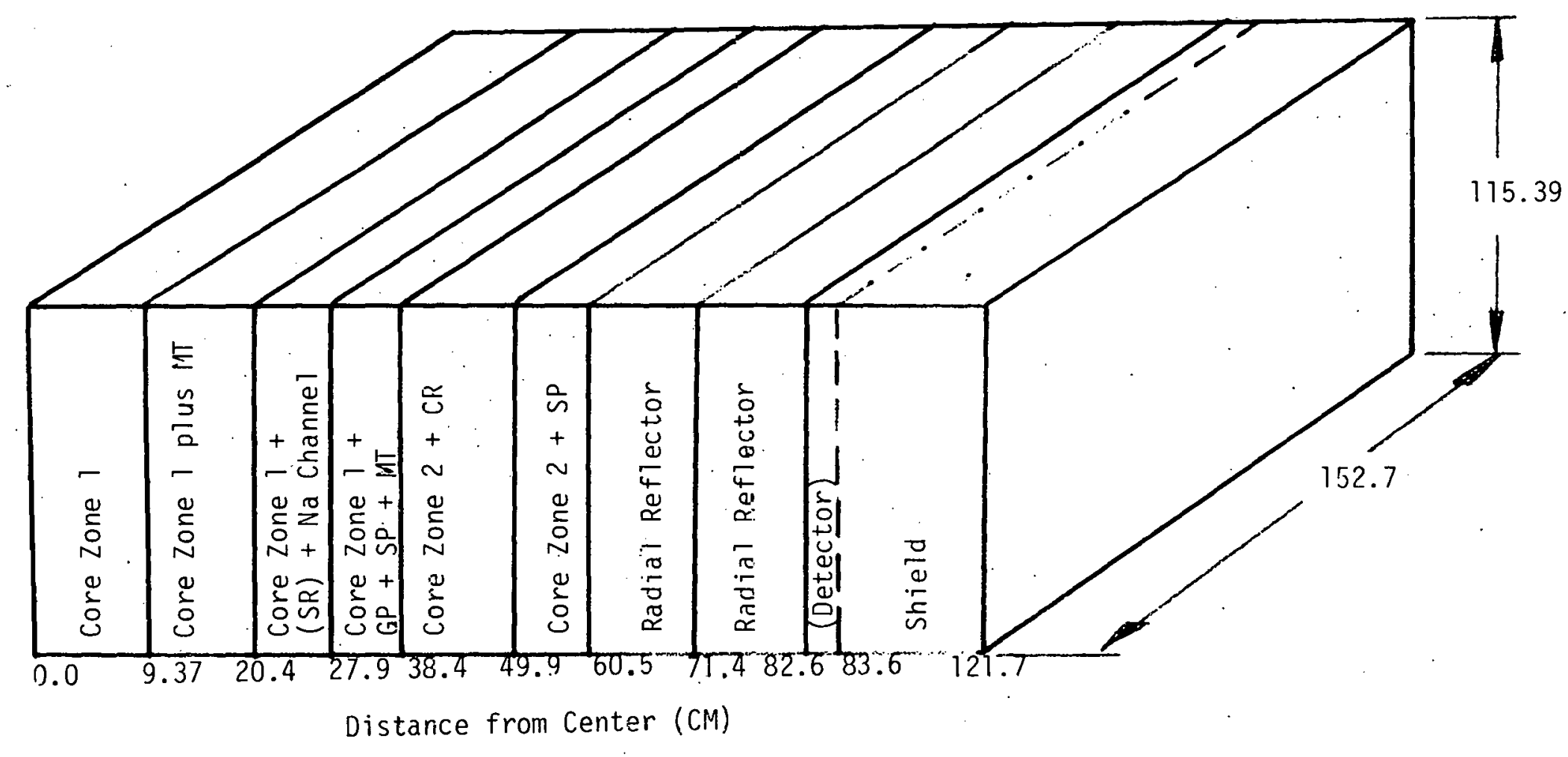

Figure 2. Slab (half) Core Configuration for Ore-Dimensional Calculations. 
PSR: Peripheral Shim Rod

CR: Control Rod

SR: Safety Rod

GP: General Purpose Loop

MT: Material Test Loop

SP: Special Purpose Loop

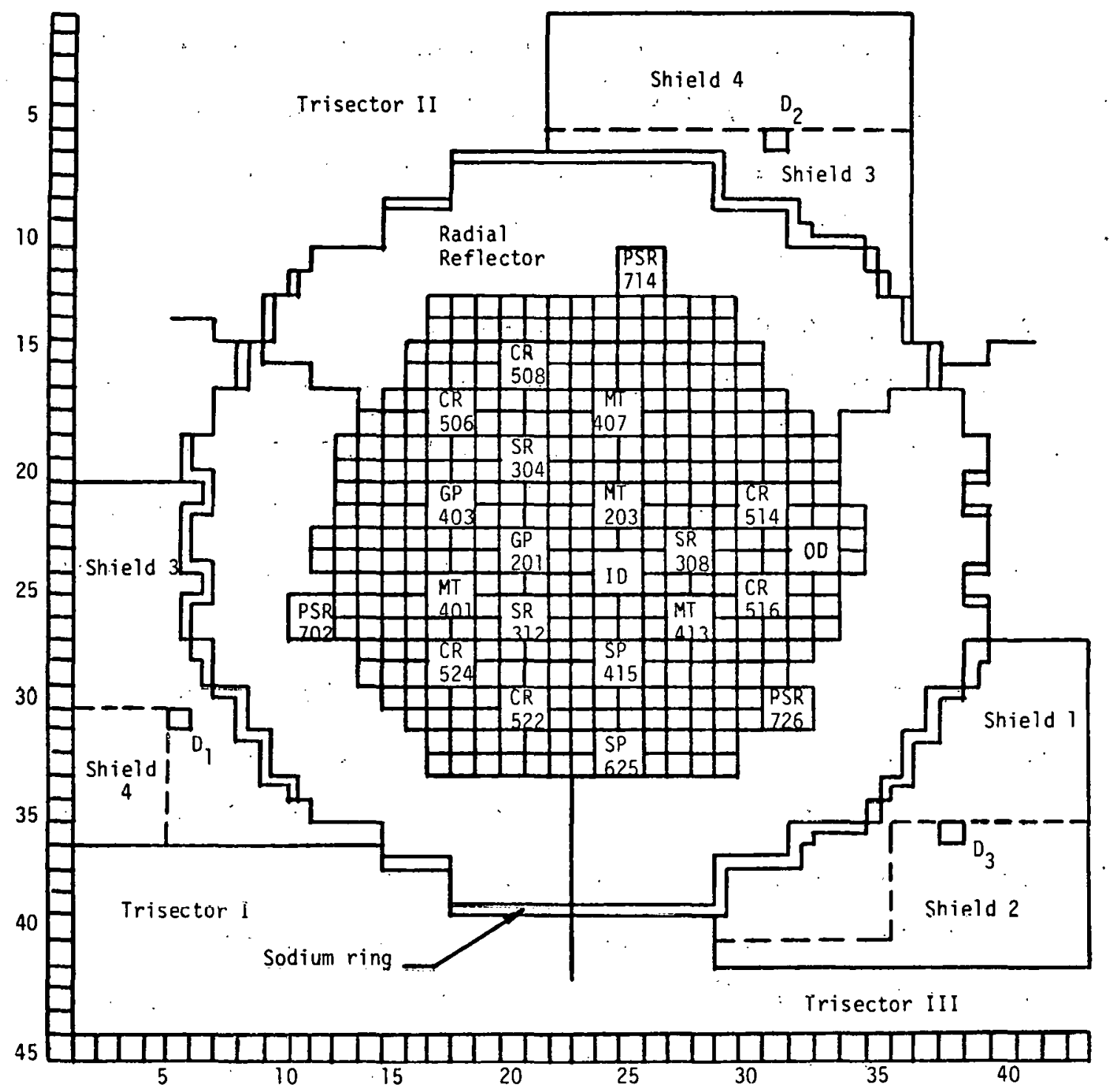

Figure 3. The EMC Core Configuration at Midplane. 
$\Delta x_{j}=$ size of the $j^{\text {th }}$ intervat,

$\phi_{g j}=$ group flux for the $j^{\text {th }}$ interval.

The dimensions of the slab core and the arrangement of material zones as well as the detector position are shown in Figure.2. The value of $\bar{v}$ is 2.92 neutrons/fission for the 50 -group EMC calculation. (33) The reactor core was divided into 18 zones. The total number of intervals is 102 , five intervals for each fueled zone, and seven intervals for each reflector and shield zone. The forward and adjoint fluxes were calculated by $\operatorname{ANISN}^{(10)}$ with $\mathrm{P}_{3} \mathrm{~S}_{4}$. The neutron crosssection set used is the 50 -group set $(45,46)$ prepared for the EMC calculation.

Reference States and Reference-flux Calculation

For variational estimations, there are four core configurations chusen as the reference states for the calculations of the reference forward and/or adjoint flux function; these states are numbered 2, 5,8 , and 11 in Table 2, which also shows the subcriticality of each state.

In order to make numerical demonstrations, the forward reference fluxes were calculated for all reference states while that for the adjoints were calculated for states 5 and 11 only. The specification of the fixed source $\bar{S}$ for the forward flux in Equation (2.10) was made by considering mainly ${ }^{(19)}$ the number density distribution of ${ }^{240} \mathrm{Pu}$ for the spontaneous fission neutrons in fast reactors. For the calculations of the adjoint fluxes $\bar{\theta}_{1}$ * and $\bar{\theta}_{2}$ * in Equations (2.11) and (2.12), the source specifications were made according to the distributions of the macroscopic detection 
TABLE 2

STATES AND SUBCRITICALITIES FOR ONE-DIMENSIONAL CALCULATIONS

\begin{tabular}{|c|c|c|c|c|}
\hline \multirow{2}{*}{$\begin{array}{l}\text { Number of } \\
\text { Control Rods } \\
\text { Inserted }\end{array}$} & \multicolumn{4}{|c|}{ Number of Safety Rods Inserted } \\
\hline & 0 & 1 & 2 & 3 \\
\hline 4 & $1.0(0.0 \$)$ & $\begin{array}{c}(4) \\
0.9661(10.55 \$)\end{array}$ & $0.9412(18.31 \$)$ & $\begin{array}{c}(10) \\
0.9199(24.9 \$)\end{array}$ \\
\hline 5 & $\begin{array}{c}(2) \\
0.9913(2.71 \$)\end{array}$ & $0.9572(13.33 \$)$ & $0.9353(20.14 \$)$ & $\begin{array}{c}(11) \\
0.9146(26.57)\end{array}$ \\
\hline 6 & $\begin{array}{c}(3) \\
0.9817(5.68 \$)\end{array}$ & $\begin{array}{c}(6) \\
0.9491(15.86 \$)\end{array}$ & $0.9264(22.9 \$)$ & $\begin{array}{c}(12) \\
0.9037(29.96 \$)\end{array}$ \\
\hline
\end{tabular}

${ }^{a}(n)$ the number " $n$ " inside the bracket represents the state number. 
cross section $\bar{\Sigma}_{d}$, and the macroscopic fission cross section $\bar{\Sigma}_{f}$ in the detector interval and the fueled reactor zones respectively.

Variational Calculational Methodology

The numerical calculation for the conventional variational method was done by choosing the reference fluxes (forward and adjoint) at the same state. We chose states 5 and 11 for this purpose. The computation is done with Equation (2.20), where the two correction terms, $\left\langle\bar{\theta}^{\star}{ }_{11}, \mathrm{~S}-\mathrm{H}_{\bar{\phi}}\right\rangle$ and $\left\langle\bar{\theta}^{\star}{ }_{21}, \mathrm{~S}-\mathrm{H}_{\bar{\phi}}\right\rangle$, for the numerator and denominator respectively, were evaluated by SWANLAKE. (3)

For the variational interpolation and extrapolation methods, the estimations of the neutron detection efficiency are done by Equation (2.24) and Equation (2.28) respectively. The selections of reference states for the two methods are similar; for the varlational interpolation, we always keep the perturbed state inside the two reference states chosen, while that for the variational extrapolation is outside. The reference-state couples used for these calculations are $\left(2,5^{\star}\right),{ }^{\mathrm{a}}\left(2,11^{*}\right),\left(8,5^{*}\right),\left(8,11^{*}\right),\left(5,11^{*}\right)$, and $\left(11,5^{\star}\right)$. The calculations of the correction terms are similar to that for the conventional variational method.

For the multi-reference-state method, the trial flux functions (forward and adjoint) are the linear combinations of given reference fluxes. There are two kinds of combinations for the trial flux

${ }^{a}\left(2,5^{*}\right)$ means that we use forward reference flux at state 2 , and adjoint reference flux at state 5 . 
functions in this calculation. The first chose the forward fluxes from states 2 and 8 as components of Equation (2.29), and that for the adjoint fluxes we selected states 5 and 11 as components of Equation (2.30). The second used the forward and adjoint fluxes from states 5 and 11 as components of Equations (2.29) and (2.30). From Equations (2.38) and (2.40), we know that the elements of the matrix are $<\Sigma_{\ell}, \bar{\phi}_{j}>,\left\langle\bar{\theta}_{\ell j}, S>\right.$, and $\left\langle\bar{\theta}_{\ell}^{\star}{ }_{\ell j}, \Delta H_{\bar{\phi}}>\cdot<\Sigma_{\ell}, \bar{\phi}_{j}>\right.$ was calculated by ANISN directly; $\left\langle\bar{\theta}_{. \ell j}\right.$, S> was computed by using the adjoint flux and the fixed source (see Appendix $E$ ); $\left\langle\bar{\theta}{ }_{\ell j}, \Delta H_{\bar{\phi}} \bar{j}\right.$ is evaluated by the SWANLAKE code (see Appendix D).

\section{$\underline{\text { Results }}$}

The detection efficiencies for a U-235 fission chamber and a He-3 detector calculated by ANISN for different states are shown in Table 3. The detection efficiencies estimated by the conventional variational method are shown in Tables 4 and 5 for U-235 and He-3 detectors respectively. Tables 6 and 7 show the values evaluated by the variational interpolational methods for $\mathrm{U}-235$ and $\mathrm{He}-3$ detectors respectively, while that computed by the variational extrapolational method are shown in Tables 8 and 9 . The multireference-state variational methods estimated detection efficiencies for the fission chamber and the $\mathrm{He}-3$ detector are shown in Tables 10 and 11 .

\section{Discussion of Results}

The reference forward and adjoint fluxes for the one-dimensional variational calculations were calculated by $\operatorname{ANISN}^{(10)}$ using 10 and 
TABLE 3

THE DETECTION EFFICIENCY OF U-235

AND He-3 DETECTOR AT EACH STATE

BY ONE-DIMENSIONAL CALCULATION

\begin{tabular}{ccc}
\hline $\begin{array}{c}\text { State } \\
\text { Number }\end{array}$ & U-235 & Detection Efficiency \\
\hline 2 & $5.51-7^{\mathrm{a}}$ & $1.84-4$ \\
3 & $5.37-7$ & $1.79-4$ \\
4 & $6.07-7$ & $2.01-4$ \\
5 & $5.92-7$ & $1.97-4$ \\
6 & $5.79-7$ & $1.92-4$ \\
7 & $6.36-7$ & $2.12-4$ \\
8 & $6.26-7$ & $2.08-4$ \\
9 & $6.12-7$ & $2.04-4$ \\
10 & $6.65-7$ & $2.21-4$ \\
11 & $6.55-7$ & $2.18-4$ \\
12 & $6.42-7$ & $2.13-4$ \\
. & & \\
\hline \hline
\end{tabular}

$a_{\text {Read }} 5.51 \times 10^{-7}$. 
TABLE 4

THE DETECTION EFFICIENCY OF U-235 DETECTOR

BY CONVENTIONAL VARIATIONAL METHOD

FOR ONE-DIMENSIONAL CORE

\begin{tabular}{ccc}
\hline $\begin{array}{c}\text { Perturbation } \\
\text { State }\end{array}$ & $\begin{array}{c}\text { Detection } \\
\text { Efficiency }\end{array}$ & $\begin{array}{c}\text { Error } \\
(\%)\end{array}$ \\
\hline$\left(5,5^{*}\right) \rightarrow 2$ & $5.54-7$ & $+0.67^{*}$ \\
$\left(5,5^{*}\right) \rightarrow 3$ & $5.45-7$ & +1.52 \\
$\left(5,5^{*}\right) \rightarrow 4$ & $5.96-7$ & -1.10 \\
$\left(5,5^{*}\right) \rightarrow 6$ & $5.86-7$ & +1.18 \\
$\left(5,5^{*}\right) \rightarrow 7$ & $7.36-7$ & +15.8 \\
$\left(11,11^{*}\right) \rightarrow 3$ & $5.89-7$ & +9.62 \\
$\left(11,11^{*}\right) \rightarrow 4$ & $6.20-7$ & +3.00 \\
$\left(11,11^{*}\right) \rightarrow 9$ & $6.17-7$ & +0.76 \\
$\left(11,11^{*}\right) \rightarrow 10$ & $6.64-7$ & -0.11 \\
$\left(11,11^{*}\right) \rightarrow 12$ & $6.43-7$ & +0.23 \\
\hline
\end{tabular}
relation

*Errors were computed from more precise values, and used the error $\%=\left(\frac{\text { exact }- \text { approximate }}{\text { exact }}\right) \times 100$. 
TABLE 5

THE DETECTION EFFICIENCY OF He-3 DETECTOR BY CONVENTIONAL V VARIATIONAL METHOD

FOR ONE-DIMENSIONAL CORE

\begin{tabular}{ccc}
\hline $\begin{array}{c}\text { Perturbation } \\
\text { State }\end{array}$ & $\begin{array}{c}\text { Detection } \\
\text { Efficiency }\end{array}$ & $\begin{array}{c}\text { Error } \\
(\%)\end{array}$ \\
\hline$\left(5,5^{*}\right) \rightarrow 2$ & $1.80-4$ & -1.92 \\
$\left(5,5^{*}\right) \rightarrow 3$ & $1.78-4$ & -0.69 \\
$\left(5,5^{*}\right) \rightarrow 4$ & $1.97-4$ & -1.93 \\
$\left(5,5^{*}\right) \rightarrow 6$ & $1.98-4$ & +2.79 \\
$\left(5,5^{*}\right) \rightarrow 7$ & $2.58-4$ & +21.7 \\
$\left(11,11^{*}\right) \rightarrow 3$ & $1.95-4$ & +8.72 \\
$\left(11,11^{*}\right) \rightarrow 4$ & $2.06-4$ & +2.37 \\
$\left(11,11^{*}\right) \rightarrow 6$ & $1.99-4$ & +3.26 \\
$\left(11,11^{*}\right) \times 8$ & $2.09-4$ & +0.12 \\
$\left(11,11^{*}\right) \rightarrow 9$ & $2.08-4$ & +1.93 \\
$\left(11,11^{*}\right) \rightarrow 10$ & $2.21-4$ & +0.10 \\
$\left(11,11^{*}\right) \rightarrow 12$ & $2.14-4$ & +0.49 \\
\hline
\end{tabular}


$\because$ TABLE 6

THE DETECTION EFFICIENCY OF U-235 DETECTOR BY VARIAT IONAL INTERPOLATION FOR

ONE-DIMENSIONAL CORE

\begin{tabular}{|c|c|c|}
\hline $\begin{array}{c}\text { Perturbation } \\
\text { State }\end{array}$ & $\begin{array}{l}\text { Detection } \\
\text { Efficiency }\end{array}$ & $\begin{array}{c}\text { Error } \\
(\%)\end{array}$ \\
\hline$\left(2,5^{\star}\right) \rightarrow 3^{\mathrm{a}}$ & $5: 33-7^{b}$ & $-0: 75^{c}$ \\
\hline$\left(2,5^{\star}\right) \rightarrow 4$. & $6.77-7$ & +12.26 \\
\hline$(2,11 *) \rightarrow 3$ & $5.28-7$ & -1.75 \\
\hline$\left(2,11^{\star}\right) \rightarrow 4$ & $5.88-7$ & -2.44 \\
\hline$(2,11 *) \rightarrow 5$ & $5.63-7$ & -4.83 \\
\hline$\left(2,11^{*}\right)+6$ & $5.30-7$ & -8.33 \\
\hline$(2,11 *) \rightarrow 7$ & $6.29-7$ & -1.19 \\
\hline$(2,11 *) \rightarrow 8$ & $5.95-7$ & -4.89 \\
\hline$(2,11 *) \rightarrow 9$ & $5.12-7$ & -16.40 \\
\hline$\left(8,5^{\star}\right) \rightarrow 6$ & $5.70-7$ & -1.44 \\
\hline$\left(8,5^{\star}\right) \rightarrow 7$ & $6.23-7$ & -2.03 \\
\hline$\left(8,11^{*}\right) \rightarrow 9$ & $6.11-7$ & -0.29 \\
\hline$\left(8,11^{*}\right) \rightarrow 10$ & $6.75-7$ & +1.44 \\
\hline$(5,11 *) \rightarrow 6$ & $5.73-7$ & -1.02 \\
\hline$(5,11 *) \rightarrow 7$ & $6.37-7$ & +0.10 \\
\hline$(5,11 *) \rightarrow 8$ & $6.19-7$ & -1.03 \\
\hline$(5,11 *) \rightarrow 9$ & $5.96-7$ & -2.65 \\
\hline$\left(11,5^{*}\right) \rightarrow 6$ & $5.65-7$ & -2.34 \\
\hline$\left(11,5^{\star}\right) \rightarrow 7$ & $5.98-7$ & -10.14 \\
\hline
\end{tabular}


TABLE 6 (Continued)

\begin{tabular}{ccc}
\hline $\begin{array}{c}\text { Perturbation } \\
\text { State }\end{array}$ & $\begin{array}{c}\text { Detection } \\
\text { Efficiency }\end{array}$ & $\begin{array}{c}\text { Error } \\
(\%)\end{array}$ \\
\hline$\left(11,5^{\star}\right) \rightarrow 8$ & $5.93-7$ & -5.24 \\
$\left(11,5^{*}\right) \rightarrow 9$ & $5.93-7$ & -3.14 \\
$\left(11,5^{*}\right) \rightarrow 10$ & $6.47-7$ & -2.75 \\
\hline
\end{tabular}

${ }^{a}$ This means that the correction term is calculated by the forward flux at State 2 and the adjoint fluxes at State 5 for the perturbation State 3 .

$b_{\text {Read }} 5.33 \times 10^{-7}$.

$c$ "+" or "-" means over or under estimation. 
TABLE 7

THE DETECTION EFFICIENCY OF He-3 DETECTOR BY VARIATIONAL INTERPOLATION

FOR ONE-DIMENSIONAL CORE

\begin{tabular}{|c|c|c|}
\hline $\begin{array}{c}\text { Perturbation } \\
\text { State }\end{array}$ & $\begin{array}{l}\text { Detection } \\
\text { Efficiency }\end{array}$ & $\begin{array}{c}\text { Error } \\
(\%)\end{array}$ \\
\hline$\left(2,5^{\star}\right) \rightarrow 3$ & $1.81-4$ & +0.90 \\
\hline$\left(2,5^{\star}\right) \rightarrow 4$ & $2.42-4$ & +20.6 \\
\hline$\left(2,11^{\star}\right) \rightarrow 3$ & $1.76-4$ & -1.49 \\
\hline$(2,11 *) \rightarrow 4$ & $1.97-4$ & -1.76 \\
\hline$(2,11 *) \rightarrow 5$ & $1.89-4$ & -3.96 \\
\hline$\left(2,11^{*}\right) \rightarrow 6$ & $1.79-4$ & -7.00 \\
\hline$\left(2,11^{\star}\right) \rightarrow 7$ & $2.13-4$ & +0.86 \\
\hline$\left(2,11^{*}\right) \rightarrow 8$ & $2.04-4$ & -2.15 \\
\hline$(2,11 *) \rightarrow 9$ & $1.87-4$ & -8.00 \\
\hline$\left(8,5^{\star}\right) \rightarrow 6$ & $1.86-4$ & -3.28 \\
\hline$\left(8,5^{\star}\right) \rightarrow 7$ & $2.06-4$ & -2.80 \\
\hline$\left(8,11^{*}\right) \rightarrow 9$ & $2.03-4$ & -0.10 \\
\hline$(8,11 *) \rightarrow 10$ & $2.25-4$ & +1.90 \\
\hline$(5,11 *) \rightarrow 6$ & 1.91-4 & -0.75 \\
\hline$(5,11 *)+7$ & $2.13-4$ & -0.64 \\
\hline$\left(5,11^{\star}\right) \rightarrow 8$ & $2.08-4$ & -0.33 \\
\hline$(5,1[*) \rightarrow y$ & $2.00-4$ & -1.62 \\
\hline$\left(11,5^{\star}\right) \rightarrow 6$ & $1.82-4$ & -5.30 \\
\hline
\end{tabular}


46

TABLE 7 (Continued)

\begin{tabular}{ccc}
\hline $\begin{array}{c}\text { Perturbation } \\
\text { State }\end{array}$ & $\begin{array}{c}\text { Detection } \\
\text { Efficiency }\end{array}$ & $\begin{array}{c}\text { Error } \\
(\%)\end{array}$ \\
\hline$\left(11,5^{*}\right) \rightarrow 7$ & $1.94-4$ & -8.31 \\
$\left(11,5^{*}\right) \rightarrow 8$ & $1.94-4$ & -6.86 \\
$\left(11,5^{*}\right) \rightarrow 9$ & $1.94-4$ & -4.75 \\
$\left(11,5^{*}\right) \rightarrow 10$ & $2.13-4$ & -3.48 \\
\hline
\end{tabular}


TABLE 8

THE DETECTION EFFICIENCY OF U-235 DETECTOR

BY VARIAT:IONAL : EXTRAPOLATION

FOR ONE-DIMENSIONAL CORE

\begin{tabular}{|c|c|c|}
\hline $\begin{array}{c}\text { Perturbation } \\
\text { State } \\
\end{array}$ & $\begin{array}{l}\text { Detection } \\
\text { Efficiency }\end{array}$ & $\begin{array}{c}\text { Error } \\
(\%)\end{array}$ \\
\hline$\left(2,5^{\star}\right) \rightarrow 7$ & $3.11-7$ & -51.1 \\
\hline$\left(2,5^{\star}\right) \rightarrow 8$ & $4.22-7$ & -32.6 \\
\hline$\left(2,5^{\star}\right) \rightarrow 9$ & $4.73-7$ & -22.7 \\
\hline$\left(2,5^{\star}\right) \rightarrow 10$ & $4.40-7$ & -33.7 \\
\hline$\left(8,5^{\star}\right) \rightarrow 2$ & $5.55-7$ & +0.78 \\
\hline$\left(8,5^{\star}\right) \rightarrow 3$ & $5.99-7$ & +11.48 \\
\hline$\left(8,5^{\star}\right) \rightarrow 4$ & $5.80-7$ & -3.68 \\
\hline$\left(8,5^{\star}\right) \rightarrow 9$ & $6.30-7$ & +2.86 \\
\hline$\left(8,5^{\star}\right) \rightarrow 10$ & $7.75-7$ & +16.6 \\
\hline$\left(8,5^{\star}\right) \rightarrow 11$ & $8.94-7$ & +36.6 \\
\hline$\left(8,11^{\star}\right) \rightarrow 2$ & $5.94-7$ & +7.18 \\
\hline$\left(8,11^{*}\right) \rightarrow 3$ & $5.78-7$ & +7.65 \\
\hline$\left(0,11^{*}\right) \rightarrow 4$ & $0.21-7$ & +3.06 \\
\hline$\left(8,11^{\star}\right) \rightarrow 5$ & $6.04-7$ & +2.10 \\
\hline$(8,1.1 *) \rightarrow 6$ & $5.91-7$ & +2.06 \\
\hline$\left(8,11^{\star}\right) \rightarrow 7$ & $6.38-7$ & +3.00 \\
\hline$\left(8,1^{*}\right) \rightarrow 12$ & $6.48-7$ & +0.90 \\
\hline$\left(11,5^{\star}\right) \rightarrow 4$ & $5.74-7$ & -4.80 \\
\hline
\end{tabular}


TABLE 8 (Continued)

\begin{tabular}{ccc}
\hline $\begin{array}{c}\text { Perturbation } \\
\text { State }\end{array}$ & $\begin{array}{c}\text { Detection } \\
\text { Efficiency }\end{array}$ & $\begin{array}{c}\text { Error } \\
(\%)\end{array}$ \\
\hline$\left(11,5^{*}\right) \rightarrow 12$ & $6.68-7$ & +4.11 \\
$\left(5,11^{*}\right) \rightarrow 2$ & $5.77-7$ & +4.70 \\
$\left(5,11^{*}\right) \rightarrow 3$ & $5.61-7$ & +4.43 \\
$\left(5,11^{*}\right) \rightarrow 4$ & $6.07-7$ & +0.80 \\
\hline
\end{tabular}


TABLE 9 .

THE DETECTION EFFICIENCY OF He-3 DETECTOR BY VARIATIONAL. EXTRAPOLATION: FOR

ONE-DIMENSIONAL CORE

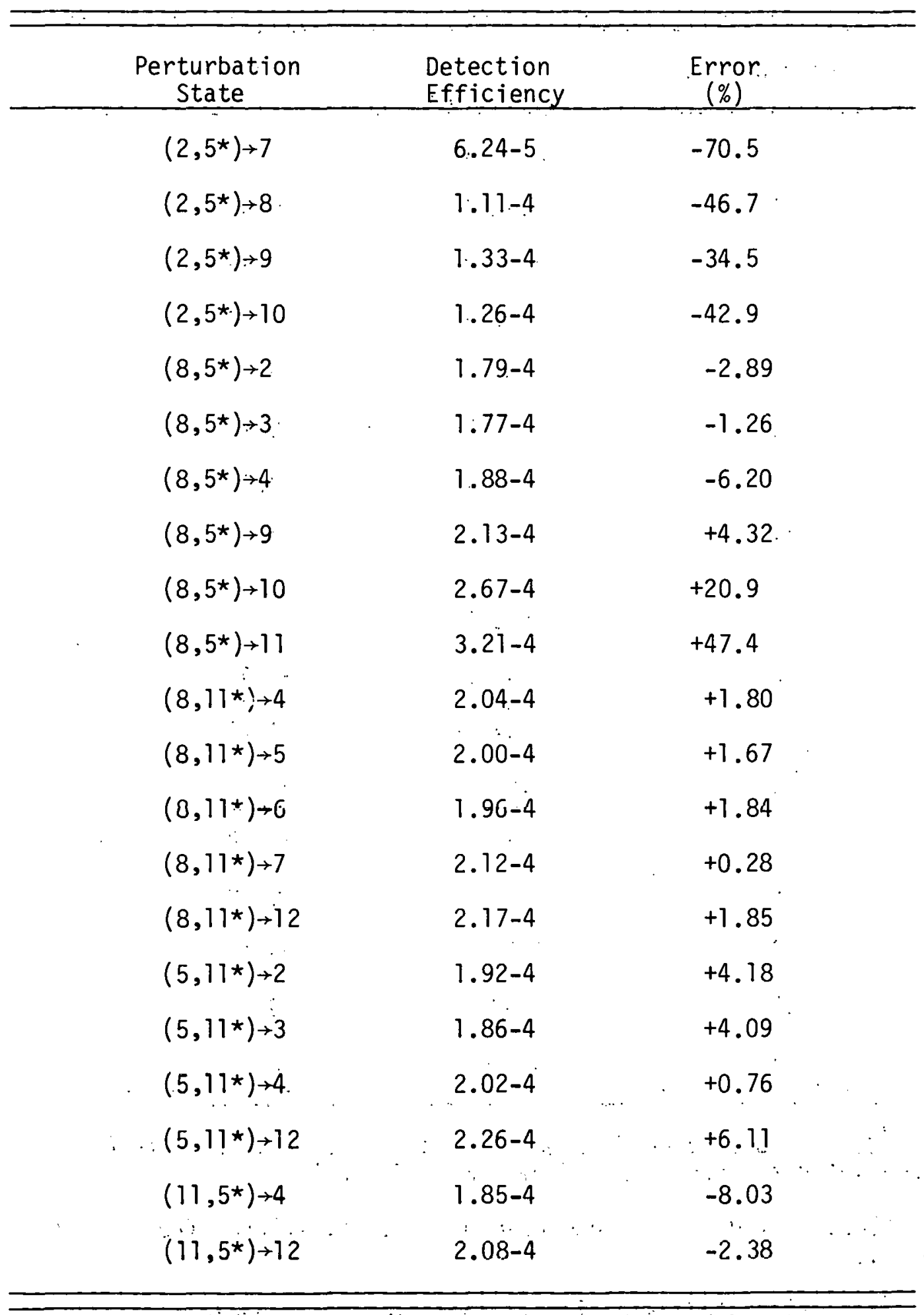


TABLE 10

THE DETECTION EFFICIENCY OF U-235 DETECTOR

BY MULTI-REFERENCE-STATE VARIATIONAL

METHOD FOR ONE-DIMENSIONAL CORE

\begin{tabular}{|c|c|c|}
\hline $\begin{array}{c}\text { Perturbation } \\
\text { State }\end{array}$ & $\begin{array}{l}\text { Detection } \\
\text { Efficiency }\end{array}$ & $\begin{array}{c}\text { Error } \\
(\%)\end{array}$ \\
\hline & Case $A^{a}$ & \\
\hline 3 & $5.42-7$ & +0.93 \\
\hline 4 & $6.05-7$ & -0.44 \\
\hline 6 & $5.80-7$ & +0.20 \\
\hline 7 & $6.40-7$ & +0.60 \\
\hline 9 & $6.14-7$ & +0.24 \\
\hline 10 & $6.71-7$ & +0.86 \\
\hline 12 & $6.42-7^{*}$ & +0.01 \\
\hline & Case $B^{b}$ & \\
\hline 3 & $5.42-7$ & +0.95 \\
\hline 4 & $6.07-7^{\star}$ & +0.78 \\
\hline 6 & $5.88-7$ & +1.55 \\
\hline 7 & $6.39-7$ & +0.49 \\
\hline 8 & $6.25-7$ & -0.09 \\
\hline 9 & $6.13-7$ & +0.16 \\
\hline 10 & $6.69-7$ & +0.54 \\
\hline 12 & $6.43-7$ & +0.19 \\
\hline
\end{tabular}

${ }^{a}$ Case $A$ used forward reference-fluxes from States 2 and 8 , and adjoint reference-fluxes from States 5 and 11 .

${ }^{b}$ Case $B$ used forward and adjoint reference-fluxes from States 5 and 11.

* The calculated value is five position after decimal point. 
TABLE 11

THE DETECTION EFFICIENCY OF He-3 DETECTOR

BY MULT I -REFERENCE STATE. VARIATIONAL

METHOD FOR ONE-DIMENSIONAL CORE

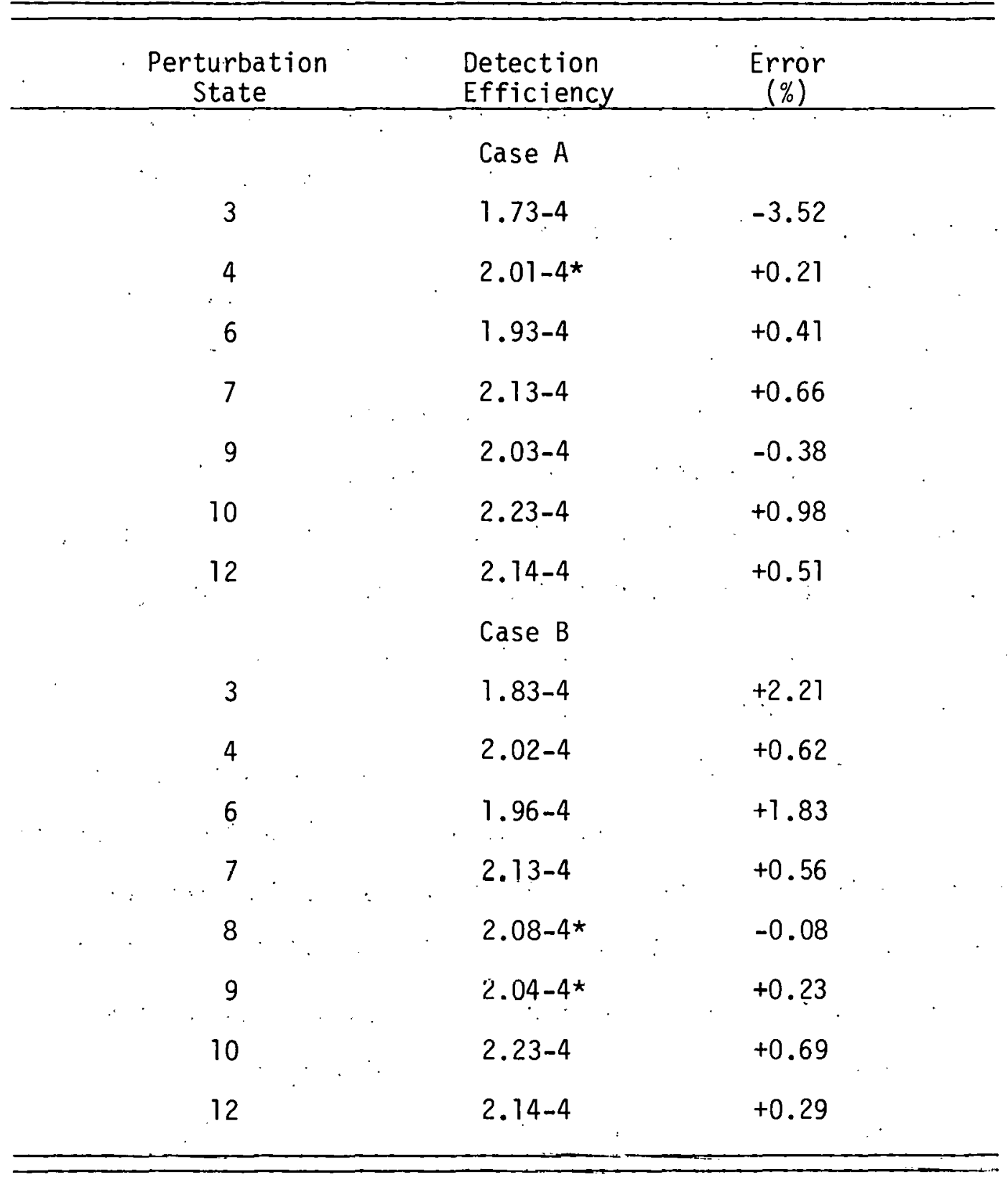

*The calculated value is five position after the decimal point. 
16 outer iterations respectively. The number of outer iterations for states like 2 and 5 was still not sufficient to have a convergent neutron flux distribution. The effect of this nonconvergence will be noted below.

Detection efficiencies for reference states and perturbed states were calculated by ANISN and are presented in Table 3. These values were used for comparison with those estimated by the variational method proposed in this dissertation.

The results for the conventional variational method (shown in Tables 4 and 5) indicate that when the perturbed state, the state to be estimated, is far from the reference-state (e.g., state 5 or state 11), the error becomes larger. For example, in Tables 4 and 5 , the discrepancies for the perturbed states 3 and 4 with state 11 as reference state were larger than that for states 10 and 12 . In general, this is an expected tendence, since the application of the variational method has a limitation.

For the variational interpolational method (results shown in Tables 6 and 7), there are two observations: (a) like the conventional variational method, when the perturbed state is far from the forward reference-state (e.g., $\left(2,1^{*}\right) \rightarrow 3,4,5,6$, and $\left(11,5^{*}\right) \rightarrow 7,8,9$, 10), the estimated parameter has larger discrepancy, and (b) the values estimated by the variational method employing the adjointfluxes at state 5 were not as accurate as that at state 11; for example, the values, at perturbed states $6,7,8$, and 9 , estimated by the reference-state couples $\left(5,11^{*}\right)$ were more accurate than that 
estimated from $\left(11,5^{*}\right)$. The reason for this is that the adjoint reference-flux at state 5 was not yet as convergent as that at state 11 .

Results for the variational extrapolational method are presented in Tables 8 and 9. To compare specifically with the conventional variational method and the variational interpolational method, the perturbed states with state number \pm 1 , or \pm 2 about a forward reference-state were considered. We note that the values for states 3 and 4 from the reference couples $\left(5,5^{*}\right)$ in Tables 4 and 5 were less in error than that from the couples $\left(5,11^{*}\right)$ in Tables 8 and 9; the values for states 9 and 10 from the couples $\left(8,11^{*}\right)$ in Tables 6 and 7 were also more accurate than that for the states 6 and 7 from the couples $\left(8,11^{*}\right)$ in Tables 8 and 9. According to those, the variational extrapolational results were not as accurate as the variational interpolational and the conventional variational results. But for cases employing the convergent reference-fluxes (both forward and adjoint), and the perturbed state not far from a forward reference-state, like that from the couples $\left(8,17^{*}\right)$ to states $4,5,6$, and 7 , and from $\left(5,17^{*}\right)$ to states 2, 3, and 4 in Tables 8 and 9, the discrepancies for the variational extrapolational results were less than $5 \%$.

The results for the multi-reference-state variational method, which used two reference-forward-fluxes and two reference adjointfluxes, are shown in Tables 10 and 11. Although the number of reference states was four for case $A$ (i.e., the forward and the adjoint trial functions were the linear combinations of the forward 
fluxes at states 2 and 8 , and the adjoint fluxes at states 5 and 11 , respectively), and two for case $B$ :(i.e., the forward and the adjoint trial functions were the linear combinations of the fluxes at states 5 and 11 ), the estimated results were within $1 \%$ of the actual results obtained directly.

The multi-reference-state variational method is reasonably insensitivity of the reference state fluxes. This is shown by the calculated results (see Tables 10 and 11) for the states far from the reference-states (e.g., 7, 8, 9, 10, and .12) which were not convergent (states 2 and 5) for forward-and adjoint-fluxes. Furthermore, results for states 3 and 6 which are near states 2 and 5 had discrepancies larger than states $7,8,9,10$, and 1.2, but in comparing with other methods in this dissertation, the effects of nonconvergent reference-fluxes on the multi-reference-state variational results are minimal.

From the results discussed above, we may draw the conclusions that (1) the Lewins' functional, Equation (2.13), is workable for the estimation of the neutron detection efficiency in the onedimensional problem, and (2) among the methods considered, the multireference-state variational method is the most promising with the variational interpolational method and the conventional variational method as candidates for scoping applications. 


\section{C.: Two-Dimensional Calculations}

Description

The procedures for the calculation of the neutron detection efficiency for two-dimensional problems are similar to that for the one-dimensional problem treated in Section B of this chapter. The core model for the two-dimensional study is shown in Figure 3 , page 35. The numerical formula of the detection efficiency is of the following form.

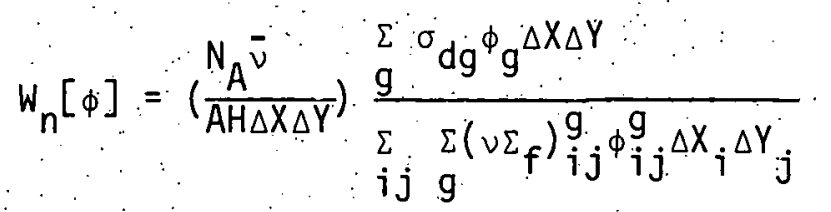

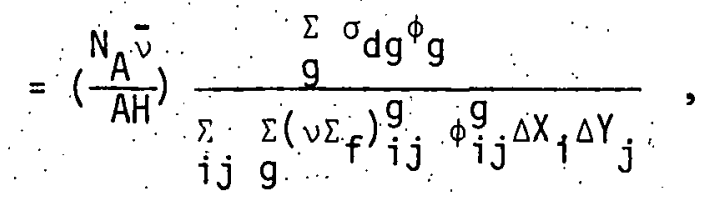

where $\Delta X_{j}$ and $\Delta Y_{j}$ are the sizes of the $i^{\text {th }}$ radial* interval and the $j^{\text {th }}$ axial* interval, respectively, other notations were described in Section $B$.

The filuxes were calculated by the two-dimensional discrete ordinates code $D O T^{(31)}$ using $\mathrm{P}_{3} \mathrm{~S}_{4}$ with 14 neutron groups (shown in Table 12). The 14-group cross sections were collapsed from the 50group cross section sets $(45,46)$ for EMC by the one-dimensional code ANISN. (10)

*According to the DOT manual, (31) the radial and axial directions represent the $X$ - and $Y$-directions, respectively. 
TABLE 12

14-ENERGY GROUP STRUCTURE AND FISSION SPECTRUM

\begin{tabular}{|c|c|c|c|c|c|}
\hline Group & & $\begin{array}{c}\text { Low Energy } \\
\text { Boundary (ev) }\end{array}$ & & $\therefore \begin{array}{r}\text { Fission } \\
\text { Spectrum }\end{array}$ & \\
\hline$\because 1$ & $\because$ & $3.07+6^{a}$ & & 0.2105 & \\
\hline 2 & & $1.35+6$ & & 0.3650 & $\therefore$ \\
\hline 3 & & $9.07+5$ & & 0.1487 & \\
\hline 4 & & $4.08+5$ & & 0.1752 & \\
\hline 5 & & $1.23+5$ & & 0.0632 & \\
\hline 6 & & $1: 93+4$ & '. & 0.0167 & \\
\hline 7 & & $3.35+3$ & & 0 & \\
\hline 8 & & $9.67+2$ & & 0 & \\
\hline 9 & & $1: 01+2$ & & 0 & \\
\hline 10 & & $2.26+1$ & . & 0 & \\
\hline 11 & & $1.07+1$ & & 0 & \\
\hline 12 & & $5.04+0$ & & 0 & \\
\hline 13. & & $1.13+0$ & & 0 & \\
\hline 14 & & Therma 1 & . & 0 & \\
\hline
\end{tabular}

$a_{\text {Read }} 3: 01 \times 10^{6}$. 
In searching for the critical core configuration, energy- and zone-dependent buckling corrections were considered ${ }^{(24)}$ in the direction perpendicular to the $X-Y$ plane' (i.e., the axial-direction). The resultant 14-group buckling cross section set, ${ }^{(33)}$ are presented in Table 13.

\section{Reference States and Flux Calculations}

In order to make numerical demonstrations, four reference states were selected from a one-dollar state to the full-shutdown, or the 30-doliar state. The control-rod positions for the four reference states and the perturbed states are shown in Figure 3 , page 35 , and Table 14 . Figure 3 , page $35^{\circ}$, is a two-dimensional core arrangement for EMC. The core consists of two fuel-zones (inner driver (ID) and outer driver (OD)) and three trisectors as well as three shielding-zones. The three trisectors are nearly symmetrical in configuration. In Table 14, states S1 and S3 were chosen for the calculations of the reference adjoint-flux calculations. The calculation of the forward and the adjoint fluxes were done by DOT. The specifications of the fixed sources for both the forward- and adjoint-flux calculations were similar to the one-dimensional problem, i.e., the calculation of $\bar{\theta}^{\star}{ }_{1}$ specifying $\bar{\Sigma}_{d}$ as adjoint source, and that for $\bar{\theta}^{\star}{ }_{2}$ specifying $\bar{\Sigma}_{f}$ in the fueled zones as source.

The selected reference states were symmetric for three trisectors. The purpose of this was using the fluxes at symmetric states to estimate the parameter of interest at perturbed states which were antisymmetric. 
TABLE 13

BUCKLING CROSS SECTIONS IN AXIAL DIRECTION FOR CORE ZONES AND RADIAL REFLECTOR $\left(\mathrm{cm}^{-1}\right)$

\begin{tabular}{cccc}
\hline Group & $\begin{array}{c}\text { Inner } \\
\text { Core }\end{array}$ & $\begin{array}{c}\text { Outer } \\
\text { Core }\end{array}$ & $\begin{array}{c}\text { Radial } \\
\text { Reflector }\end{array}$ \\
\hline 1 & $3.544-3^{\mathrm{a}}$ & $3.767-3$ & $3.261-3$ \\
2 & $2.946-3$ & $2.604-3$ & $1.848-3$ \\
3 & $1.772-3$ & $1.502-3$ & $1.601-3$ \\
4 & $1.455-3$ & $1.537-3$ & $7.335-4$ \\
5 & $9.509-4$ & $1.009-3$ & $5.939-4$ \\
6 & $6.233-4$ & $5.841-4$ & $4.209-4$ \\
7 & $-1.322-4$ & $5.782-5$ & $1.196-4$ \\
8 & $-6.878-4$ & $-3.645-4$ & $1.933-5$ \\
9 & $-1.684-3$ & $-2.387-3$ & $-5.935-5$ \\
10 & $-1.249-2$ & $-0.344-3$ & $1.261-5$ \\
11 & $-3.585-2$ & $-1.540-2$ & $-2.063-4$ \\
12 & $-5.369-2$ & $-1.338-2$ & $-8.795-6$ \\
13 & $-2.416-2$ & $-1.693-2$ & $-3.537-4$ \\
14 & $-2.599-1$ & $-5.315-2$ & $7.948-4$ \\
\hline
\end{tabular}

${ }^{a}$ Read $3.544 \times 10^{-3}$. 
TABLE 14

CONFIGURATIONS OF REFERENCE STATES AND PERTURBED

STTATES FOR TWO-DIMENSIONAL CALCULATIONS

\begin{tabular}{|c|c|c|c|c|c|c|c|c|c|c|c|c|c|c|c|c|}
\hline \multicolumn{3}{|c|}{ States ${ }^{\exists}$} & $\begin{array}{l}\mathrm{CR}^{\mathrm{Tr}} \\
522 \\
\end{array}$ & $\begin{array}{l}\text { isect } \\
\text { CR } \\
524 \\
\end{array}$ & $\begin{array}{l}\text { or I } \\
\text { SR } \\
312 \\
\end{array}$ & $\begin{array}{l}\text { PSR } \\
702 \\
\end{array}$ & $\begin{array}{l}\mathrm{CR}^{\mathrm{Tr}} \\
\mathrm{506} \\
\end{array}$ & $\begin{array}{c}\text { isect } \\
\text { CR } \\
508 \\
\end{array}$ & $\begin{array}{l}\text { or II } \\
\text { SR } \\
304 \\
\end{array}$ & $\begin{array}{l}\text { PSR } \\
714 \\
\end{array}$ & . & $\begin{array}{l}\text { Tri } \\
\text { CR } \\
514 \\
\end{array}$ & $\begin{array}{l}\text { secto } \\
\text { CR } \\
516 \\
\end{array}$ & $\begin{array}{l}\text { I I I } \\
\text { SR } \\
308 \\
\end{array}$ & $\begin{array}{l}\text { PSR } \\
726 \\
\end{array}$ & \\
\hline & Critical & & $W^{*}$ & $I^{*}$ & W & I & $W$ & I & W & I & . & $W$ & I & W & I. & \\
\hline & $S \cdot T$ & .. & $w$ & $c^{b}$ & $W$ & I & $W$ & C & $W$ & I & . & $W$ & $C$ & $W$ & I & . \\
\hline & $\mathrm{P} 2$ & . & W & I & W & I & I & I & $w$ & I & & $W$ & I & $W$ & I & \\
\hline & P3 & & $W$ & I & $W$ & $W$ & I & I & $W$ & I & 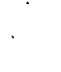 & $W$ & I & $W$ & I & \\
\hline & P4 & & I & I & W & I & I & I & $W$ & I & & $W$ & I. & $w$ & I & . \\
\hline & $\mathrm{S} 2$ & & I & I & $W$ & I & I & I & $W$ & I & . & I & I & $W$ & I & 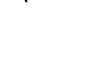 \\
\hline . & $\mathrm{P} 5$ & & I & I & $W$ & I & I & I & $W$ & $I$ & & I & I & I & I & \\
\hline & S3 & 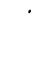 & W & I & I & I & W & I & I & I & & $W$ & $I$ & I & $\therefore \quad I$ & \\
\hline & P6 & 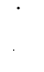 & I & I & I & I & I & I & $W$ & I & & I & I & I & I & \\
\hline & S4 & & I & I & I & I & I & I & $\mathrm{I}$ & I & . & I & I & I & I & \\
\hline
\end{tabular}

${ }^{a} S$ indicates reference state, $P$ indicates perturbed state.

boncentrated CR makes SI one dollar subcritical

*"I" is insertion, and "W" is withdrawn. 
Variational Calculational Methodology

The numerical calculation of the neutron detection efficiency by the variational methods, e.g., the variational interpolational method and the multi-reference-state variational method, were similar to the one-dimensional problem. For the variational interpolation, we chose $\left(S 1, S 2^{\star}\right),\left(S 1, S 4^{*}\right),\left(S 3, S 2^{*}\right)$, and $\left(S 3, S 4^{*}\right)$ as referencestate couples for the forward-and adjoint-fluxes. For the multireference-state variational method, we chose states S1 and S3 for the forward-fluxes for Equation (2.29), and states S2 and S4 for the adjoint-fluxes for Equation (2.30).

The calculations of the flux-change correction terms $\left\langle\bar{\theta}{ }_{1}, S-H \bar{\phi}\right\rangle$ and $\left\langle\bar{\theta}{ }_{2}, S-H \bar{\phi}\right\rangle$ in Equation (2.20) were carried out using SWANLAKE $(3)$ and a code VIP, ${ }^{(6)}$ which is employed to calculate ${ }^{(44)}$ the product of the flux moments $\bar{\theta} \star_{\phi}$ in two-dimensional problem (see Chapter III, page 30). The parameter $\left\langle\Sigma_{d} \bar{\phi}\right\rangle$ at states $S 1$ and $S 3$ were calculated by using the 14-group fission cross section set for U-235, as shown in Table 15. The value of the parameter $\left\langle\bar{\theta}{ }_{i}, S\right\rangle ; j=1$ and 2 , was computed by a program written for this need (presented in Appendix F).

Results of Two-Dimensional Calculations

The calculational results for the two-dimensional test problem are shown in Tables 16, 17, and 18. Table 16 is the directly calculated detection efficiencies for the four selected perturbed states and the two forward reference states S1 and S3. Table 17 shows the values evaluated by the variational interpolational method for detectors $D_{1}$ and $D_{3}$ (see Figure 3, page 35). Table 18 presents 
TABLE 15

14-GROUP FISSION CROSS-SECTION COLLAPSED FROM 50-GROUP SET FOR U-235

\begin{tabular}{cc}
\hline Group & $\begin{array}{c}\text { Cross-Section } \\
\text { (barn) }\end{array}$ \\
\hline 1 & $1.276+0$ \\
2 & $1.296+0$ \\
3 & $1.249+0$ \\
4 & $1.211+0$ \\
5 & $1.395+0$ \\
6 & $2.011+0$ \\
7 & $3.498+0$ \\
8 & $6.941+0$ \\
9 & $1.514+1 *$ \\
10 & $3.027+1$ \\
11 & $5.635+1$ \\
12 & $9.821+1$ \\
13 & $1.615+1$ \\
14 & $1.237+2$ \\
\hline
\end{tabular}

$\star^{\text {Read }} 1.514 \times 10^{1}$. 
TABLE 16

NEUTRON DETECTION EFFICIENCY FOR, FISSION CHAMBERS

AT $D_{1}$ AND $D_{3}$ CAL CULATED BY DOT

\begin{tabular}{|c|c|c|c|}
\hline & Case & $D_{1}$ & $\mathrm{D}_{3}$ \\
\hline & S1 & $2.894-7^{\star}$ & $1.998-7$ \\
\hline & P2 & $2.807-7$ & $2.139-7$ \\
\hline & P4 & $2.856-7$ & $2.196-7$ \\
\hline & S3 & $3.389-7$ & $2.356-7$ \\
\hline \multirow{2}{*}{ ' } & P5 & $3.498-7$ & $2.165-7$ \\
\hline & P6 & $3.468-7$ & $2.271-7$ \\
\hline
\end{tabular}

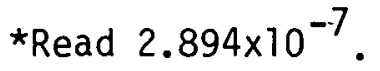


TABLE 17

TWO-DIMENSIONAL DETECTION EFFICIENCY ESTIMATED

BY VARIATIONAL INTERPOLATIONAL METHOD

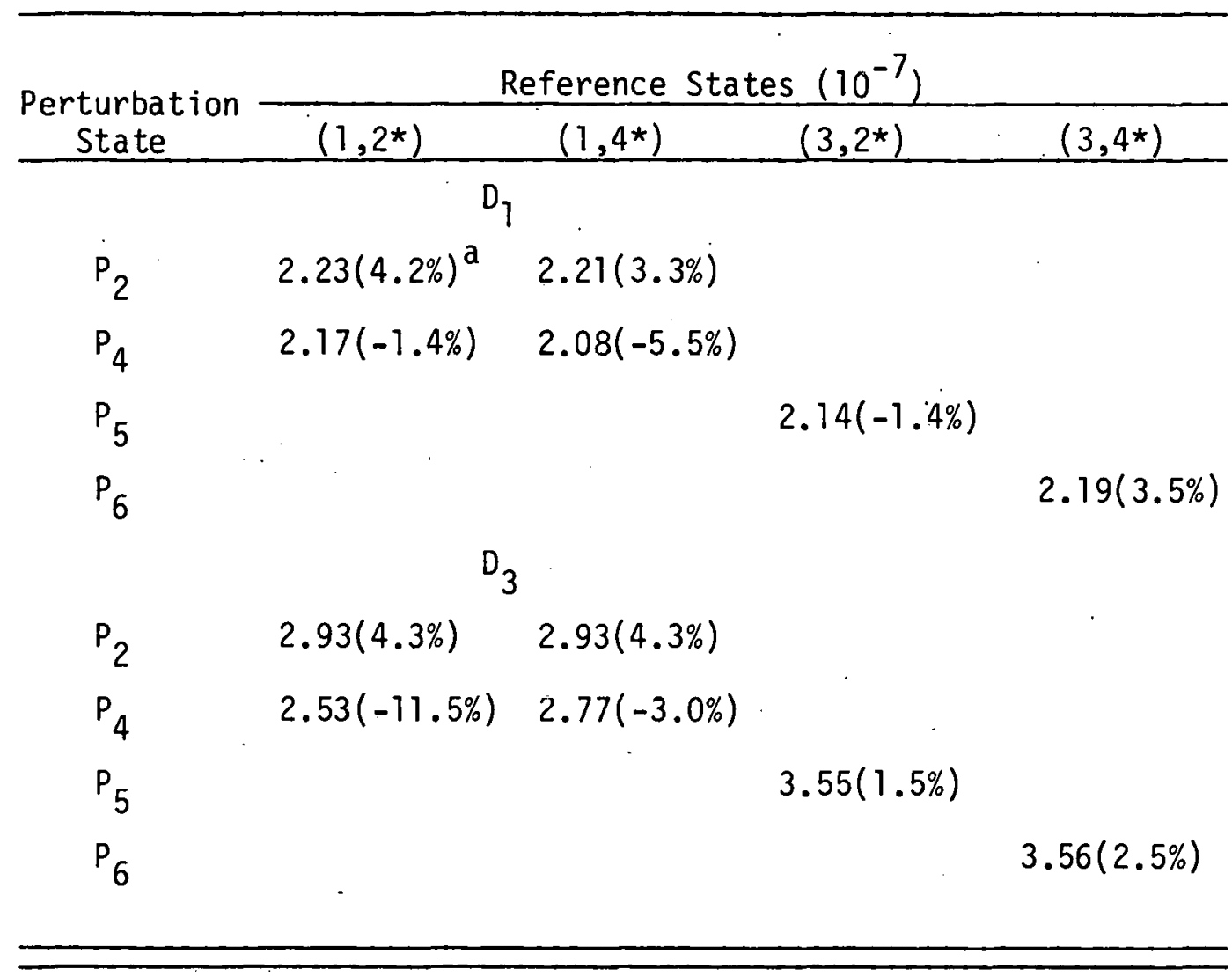

${ }_{B}$ Bracketed values are error percentages compared with direct calculation. 
TABLE 18

TWO-DIMENSIONAL DETECTION ÉFF.ICIENCY ESTIMATED BY MULTI-REFERENCE-STATE VARIATIONAL METHOD

\begin{tabular}{|c|c|c|}
\hline \multirow[b]{2}{*}{$\begin{array}{l}\text { Perturbation } \\
\text { State } \\
\end{array}$} & \multicolumn{2}{|c|}{ Detection Efficiency $\left(10^{-7}\right)$} \\
\hline & $\mathrm{D}_{1}$ & $\mathrm{D}_{3}$ \\
\hline $\mathrm{P}_{2}$ & $2.69(-4.3 \%)^{\star}$ & $2.20(2.8 \%)$ \\
\hline$P_{4}$ & $2.76(-3.4 \%)$ & $2.28(3.6 \%)$ \\
\hline$P_{5}$ & $3.66(4.6 \%)$ & $2.19(0.95 \%)$ \\
\hline $\mathrm{P}_{6}$ & $3.55(2 \%)$ & $.2 .32(2.2 \%)$ \\
\hline
\end{tabular}

*Values in brackets represent error, percentage. 
results calculated by the multi-reference-state variational method for the four perturbed states using forward fluxes from states 51 and S3, and adjoint fluxes from states $\$ 2$ and 54 .

\section{Discussion of Results}

The detection efficiencies for detectors located at $D_{1}$ and $\mathrm{D}_{3}$ are presented in Table 16, which were used for the purpose of comparison with these results calculated by the variational functional in this study. The values for $D_{1}$ are larger than that for $D_{3}$ because $D_{1}$ is close to the core zone of the EMC (see Figure 3, page 35 ). Table 17 shows the results calculated by the variational interpolational method and most were under $6 \%$ in error. For the multi-reference-state variational method, the results (calculated by use of four reference-states, two for forward fluxes and two for adjoint fluxes) are presented in Table 18, and all values were under $5 \%$ in error. Concerning these values, we note that the two-dimensional problem has a little higher error than that of the one-dimensional problem. This was partly contributed by the complication of the two-dimensional core configuration (see Figure 3, page 35) i.e., the heterogeneous distribution of the reactor material for the EMC, and partly by the concentration of the perturbation (or variation), like the moving of the control rods in this study. The heterogeneity of the core configuration increases the difficulty of reaching a convergent flux-distribution (both forward and adjoint), especially the two-dimensional adjoint-flux calculation with a point adjoint-source (at $D_{1}$ or $D_{3}$ in this study). The 
concentration of the perturbation increases the effect of the locality of the reference-fluxes at the perturbation site. The combination of the last two contributions will make an accurate estimation of the correction terms $\left\langle\bar{\theta}^{\star}{ }_{j}, S-H \bar{\phi}\right\rangle, i=1,2$, in Equation (2.13) more difficult:

Results as demonstrated in this dissertation show that both the variational interpolational method and the multi-referencestate variational method are acceptable for practical application in the estimation of the neutron detection efficiency in twodimensional problem. In particular; the multi-reference-state variational method leads to more accurate results in general when applied to a large number of perturbed states. 


\section{CHAPTER $\cdot V$}

\section{CONCLUSIONS AND RECOMMENDATIONS}

\section{A. Conclusions}

A Lewins' variational functional, ratio of two other variational functionals, has been employed in this study for the calculation of the neutron detection efficiency. Use of this formulation has been applied for two calculational models of a fast test reactor, one for the one-dimensional problems and the other for the two-dimensional problems, and shown to be an acceptable technique.

Theoretically, it has been shown that the porposed variational functional is equivalent to the conventional formulation presented in the literature based upon the first order error in neutron fluxes (forward and adjoint). The flexibility of the functional has been demonstrated by applications employing four different variational calculational methods: conventional variational, variational interpolation, variational extrapolation, and multi-reference-state variational. The results from these four approaches show that the multï-reference-state variational method is the most promising for the estimation of the neutron detection efficiency, and with proper selection of reference states as well as the size of perturbation, the conventional variational method and the variational interpolational method are acceptable for practical applications. 
The computational procedures for the suggested functional have also been developed. And following these procedures, the computation time for the functional is much less than that required by the direct calculation for a perturbed state.

\section{B. Recommendations for Further Study}

The case studies for the application of the functional presented in this dissertation for both the one-dimensional and two-dimensional problems are all perturbations with control rods changing positions, i.e., no fissile material, such as fuel depletion, is involved in the variational calculations. The inclusion of fissile material in the perturbation are possible for the suggested functionat, Equation (3.1) and hence it is recommended that these methods be employed in fuel depletion problems.

For studies of the multi-reference-state variational method by the application of: Equations. $(2.29)$ and $(2.30)$, the numbers of reference states for both the adjoint and the forward fluxes were taken to be equal. The use of. unequal numbers of reference states (7) for adjoint and forward fluxes is possible for linear functionals. Therefore, it is recommended that the use of unequal forward and adjoint reference states for the functional used herein be explored.

The calculations of neutron fluxes in this dissertation are al1 done by the discrete ordinates method for neutron transport equations. The solutions of neutron fluxes at reference states could be solved by lower-order scattering transport equations and the effect of the higher-order scattering anisotropy of neutron 
fluxes could be corrected ${ }^{(38)}$ by taking it into consideration of the correction terms in Equation (3.1). It is recommended that this procedure be explored for the purposes of being able to reduce the amount of effort required to generate the reference-state fluxes. 


\section{THIS PAGE}

\section{WAS INTENTIONALLY \\ LEFT BLANK}


THIS PAGE

\section{WAS INTENTIONALLY \\ LEFT BLANK}




\section{BIBLIOGRAPHY}

1. Ackermann, N. J., Jr., and A. R. Buhl, "LMFBR Subcriticality Measurement System Development," ORNL-CF-70-11-67, November 1970.

2. Ackermann, N. J., Jr., "Subcriticality Measurement in an LMFBR," Muclear Safety, Vol. 12, No. 6, 1971 .

3. Bartine, D. E., F. R. Mynatt, and E. M. Oblow, "SWANLAKE, A Computer Code Utilizing ANISN Radiation Transport Calculations for Cross Section Sensitivity Analys is," ORNL-TM-3809, May 1973.

4. Bell, G. I. and S. Glasstone, Nuclear Reactor Theory, Van Nostrand Reinhold Company, New Jersey, 1970.

5. Buhl, A. R. and N. J. Ackermann, Jr., "Precision of Shutdown Margin Measurement Using the Two-Frequency Reactor Noise Technique in an LMFBR," IEEE Nucl. Sci. 18(1), p. 430, 1971.

6. Childs, R. L., "VIP, A Computer Program Using Two-Dimensional Discrete Ordinate Transport Calculations for Cross-Section Sensitivity Analysis," UCCND-CSD-1 (to be published).

7. Cheng, E. T. and R. W. Conn, "Variational Interpolation: A Multipoint Perturbation Theory for Sensitivity and Synthes is Studies," Trans. of Amer. Nucl. Soc., Vol. 19, p. 172, October 1974 .

8. Conn, R. W. and W. M. Stacey, Jr., "Variational Methods for Controlled. Thermonuclear Reactor Blanket Studies," Nuclear Fusion 13, p. 185, 1973.

9. Dwivedi, S. R., "Variational Principles for Calculation of Arbitrary Ratio of Processes in Cricital Assemblies," J. of Nucl. Energy, 22, pp.-123-125, Pergamon Press, New York, $\overline{9968 .}$

10. Engle, W. W., Jr., "A User's Manual for ANISN, A One-Dimensional Discrete Ordinates Transport Code with Anisotropic Scattering," K-1693, Computer Technology Center, 1967.

11. Finlayson, B. A., The Method of Weighted Residuals and Variational Principles, Academic Press, New York, 1972.

12. Flanagan, G. F., D. B. Simpson, and A. R. Buhl, "One-Dimensional Calculations in Support of the Reactivity Surveillance Procedures for the Fast Test Reactor," ORNL-TM--4014, April 1973. 
13. Greenspan, E., "A Generalized Perturbation Theory and Variational Principle for Multiple Ratios of Linear and Bilinear Functions," Nucl. Sci. and Eng., 56, 107, 1975.

14. Grinzner, M. L., R. D. Sabin, and J. D. White, "The Analytical Determination of the Efficiency of a Neutron Sensitive Detector," ORNL-OREPS-4, August 1968.

15. Hoffman, T. J., J. C. Robinson, and P. N. Stevens, "The Adjoint Difference Method and Its Application to Deep-Penetration Radiation Transport," Nucl. Sci. Eng., 48, 179,1972.

16. Kaplan, K., "Variational Methods in Nuclear Engineering, "Adv. Nucl. Sci. and Tech., 5, 185, Academic Press, New York, $\overline{1969}$.

17. Korn, G. A. and T. M. Korn, Mathematical Handbook for Scientists and Engineers, 15.4.2, McGraw-HiTh Book Co.; New York, 1967.

18. Kryter, R. C., D. N. Fry, and D. P. Roux, "An Evaluation of the Two-Detector Cross-Correlation Technique for Shutdown Margin Measurements in Power Reactors," ORNL-4255, September 1968.

19. Kryter, R. C., N. J. Ackermann, Jr., and A. R. Buhl, "Subcriticality Measurements in FTR-3: The Homogeneous Mockup of the Fast Test Reactor," ORNL-TM-3715, February 1972.

20. Lewins, J., Importance: The Adjoint Function, Pergamon Press, New York, 1965.

21. Lewins, J., "A Variational Principle for Ratios in Critical Systems," J. of Nuc 1. Energy Parts A/B, 20, 141-143, Pergamon Press, New York, 1966.

22. Mathews, J., and 'R. L. Walker, Mathematical Methods of Physics, W. A. Benjämin, Inc., New York, 1965.

23. Mynatt, F. R., F. J. Muckenthaler, and P..N. Stevens, "Development of Two-Dimensional Discrete Ordinates Transport Theory for Radiation Shielding," CTC-INF-952, Computing Technology Center, August 1969.

24. Petrie, L. M., and N. R. Cross, "Energy- and Region-Dependent Bucklings for the FFTF Engineering Mockup Core from Monte Carlo," Trans. of Am. Nucl. Soc., 19, 348, 1974.

25. Pomraning, G. C., "Reduction of Transport Theory to Multigroup Diffusion Theory," J. Nucl. Eng., Parts A/B, 18, 497-512, Pergamon Press, 1964.

26. Pomraning, G. C., "A Variational Principle for Linear Systems," J. Soc. Indust. App. Math., 13(2), 511-519, June 1965. 
27. Pomraning, G. C., "A Derivation of Variational Principles for Inhomogeneous Equations,". Nucl: Sci. and Eng. 29, pp. 220-236, 1967.

28. Pomraning," G. C.; "The Calculation of Ratios in Critical Systems," J. Nuclear Energy, 21, pp. 285-291, 1967.

29. Rabitz, H. and R. Conn, "Variational Techniques in Scattering Theory," Physical Review A, 3rd series, Vol. 7, No. 2, p. 577, February 1973.

30. Rampolla, D. S. and W. S. Minkler, "Notes on Perturbation Theory and Variational Methods with Special Application to the Design of Nuclear Reactors," Unpublished report, Westinghouse Company.

31. Rhoades, W. A.: and F. R. Mynatt, "The DOT-III. Two-Dimensional Discrete Ordinates Transport Code," ORNL-TM-4280, September 1973.

32.: Seifritz, W., D. Stegemann, and W. Vath, "Two Detector CrossCorrelation Experiments in the Fast-Thermal Argonaut Reactor START," KFK-413, March 1966.

33. Selby, D. L., "Subcriticality Calculations in Support of the Reactivity Surveillance Procedures for the Experimental Mockup Critical Facility, unpublished report for Nuclear Engineering Practice of The University of Tennessee, January 1975.

34. Stacey, W. M., Jr., "Variational Estimates and Generalized Perturbation Theory for the Ratios of Linear and Bilinear Functionals," J. Math. Phys., 13(8), 1119, August 1972.

35. Stacey, W. M., Jr., "Variational Estimates of Reactivity Worths and Reaction Rate Ratios in Critical Nuclear Reactors," Nuc. Sci. and Eng. 48, 444-458, 1972.

36. Stacey, W. M., Jr., "An Improved Reactivity Table Model for Liquid Metal Fast Breeder Reactor Dynamics," Nucl. Sci. and Eng., 49, pp. 213-227, 1972.

37. Stacey, W. M., Jr., Variational Methods in Nuclear Reactor Physics, Academic Press, Inc., New York, 1974.

38. Stacey, W. M., Jr., "Correction for Higher-Order Scattering Anisotropy in Neutron Transport Theory," J. of Nuclear Energy, Vo1. 27, pp. 263-272, 1973.

39. Steinke, R., "Adjoint and Neutron Flux Approximations for Use in Perturbation Theory," Trans. of Amer. Nucl. Soc., Vol. 18, 1974. 
40. Smith, D. R., Variational Methods in Optimization, Prentice-Hall, Inc., Englewood Cliffs, New Jersey, 1974.

41. Taylor, A. E. and W. R. Mann. Advanced Calculus, Second Edition, Xerox College Publishing, Lexington, 1972.

42. Usachev, L. N., "Perturbation Theory for the Breeding Ratio and for Other Number Ratios Pertaining to Various Reactor Processes," J. Nucl. Energy, Parts A.B $, 18,571,1964$.

43. Yamada, K., G. K. Mar, and J. P. Mulkey, "Prediction of Neutron Sensitive Ionization Chamber Efficiency for the EBR-II," RNL-OREPS-9, July 18, 1969.

44. Childs, R. L., D. E. Bartine, and W. W. Engle, Jr., "Perturbation Theory and Sensitivity Analysis for Two-Dimensional Shielding Calculations," p. 543, Trans. of Am. Nucl. Soc., Vol. 20, May 1975.

45. Selby, D. L., "Heterogeneous Cell-Weighted 50-Group Neutron Cross Section Sets," private communication, May 1975.

46. Ford, E., "ANISN and BOLD/VENTURE Cross Sections," Internal Correspondence, Nuclear Division, Union Carbide Corporation, April 1974. 
APPENDIXES 


\section{THIS PAGE WAS INTENTIONALLY LEFT BLANK}


APPENDIX A

THE VARIATIONAL PRINCIPLE FOR A RATIO

We define a ratio of two linear functionals as:

$$
W_{n}[\phi]=\frac{\left\langle\Sigma_{1} \phi\right\rangle}{\left\langle\Sigma_{2} \phi\right\rangle},
$$

where $\Sigma_{1}, \Sigma_{2}$ represent two different properties,

$\phi$ is the solution of the following equation

$$
H_{\phi}=S \text {. }
$$

The variational method is a mathematical way by which a trial solution could be used to estimate a parameter of interest at a perturbed state. By using the concept of the Lagrange multiplier, ${ }^{(40)}$ the functional used to optimize the parameter of interest $W_{n}[\phi]$ is defined as ${ }^{(27)}$

$$
E_{p}\left[\bar{\phi}, \theta^{*}\right]=W_{n}[\bar{\phi}]+\left\langle\theta^{*}, S-H \bar{\phi}\right\rangle,
$$

where

$$
\bar{\phi}=\dot{\phi}-\delta \phi,
$$

and the second term is a flux correction term.

For Equation (A.3) to be a variational principle for estimating $W_{n}[\phi]$, the following conditions should be satisfied: (37)

(a) $E_{\eta}$ is stationary about the function $\Phi_{S}=\phi$,

(b) The stationary value of $E_{1}$ is

$$
E_{1}\left[\bar{\phi}_{s}, \theta_{s} *\right]=W_{n}[\phi]=\frac{\left\langle\Sigma_{1} \phi\right\rangle}{\left\langle\Sigma_{2} \phi\right\rangle} \text {. }
$$


From Equation (A.3), if we let

$$
\begin{aligned}
& \bar{\phi} \rightarrow \bar{\phi}+\varepsilon \delta \bar{\phi}, \\
& \theta^{*} \rightarrow \theta^{*}+\varepsilon \delta \theta^{\star},
\end{aligned}
$$

then

$$
\mathrm{E}_{1}\left[\bar{\phi}+\varepsilon \delta \bar{\phi}, \theta^{\star}+\varepsilon \delta \theta^{\star}\right]=W_{n}[\bar{\phi}+\varepsilon \delta \bar{\phi}]+\left\langle\theta^{\star}+\varepsilon \delta \theta^{\star}, S-H(\bar{\phi}+\varepsilon \delta \bar{\phi})\right\rangle,
$$

where

$\varepsilon$ is a small definite parameter.

The first variation of a functional is defined $(40)$ as

$$
\delta E_{1}=\left.\frac{\partial E_{1}}{\partial \varepsilon}\right|_{\varepsilon=0 .}
$$

From Equation (A.5), we have :

$$
\begin{gathered}
\frac{\partial E_{1}\left[\bar{\phi}+\varepsilon \delta \bar{\phi}, \theta^{*}+\varepsilon \delta \theta^{*}\right]}{\partial \varepsilon}=\frac{\partial}{\partial \varepsilon}\left(\frac{\left\langle\Sigma_{1}(\bar{\phi}+\varepsilon \delta \bar{\phi})\right\rangle}{\left\langle\Sigma_{2}(\bar{\phi}+\varepsilon \delta \bar{\phi})\right\rangle}\right)+ \\
\frac{\partial}{\partial \varepsilon}\left(\left\langle\theta^{*}+\varepsilon \delta \theta^{*}, S-H(\bar{\phi}+\varepsilon \delta \bar{\phi})\right\rangle\right) \\
=\frac{\left\langle\Sigma_{1} \delta \bar{\phi}\right\rangle\left\langle\Sigma_{2}(\bar{\phi}+\varepsilon \delta \bar{\phi})\right\rangle-\left\langle\Sigma_{1}(\bar{\phi}+\varepsilon \delta \bar{\phi})\right\rangle\left\langle\Sigma_{2} \delta \bar{\phi}\right\rangle}{\left.<\Sigma_{2}(\bar{\phi}+\varepsilon \delta \bar{\phi})\right\rangle^{2}} \\
-\left\langle\theta^{*}+\varepsilon \delta \theta^{*}, H \delta \bar{\phi}\right\rangle+\left\langle\delta \theta^{*}, S-H(\bar{\phi}+\varepsilon \delta \bar{\phi})>.\right.
\end{gathered}
$$


Thus

$$
\begin{aligned}
& \delta E_{1}\left[\bar{\phi}, \theta^{*}\right]=\left.\frac{\partial E_{l}}{\partial \varepsilon}\right|_{\varepsilon=0}=\frac{\left\langle\Sigma_{1} \delta \bar{\phi}\right\rangle\left\langle\Sigma_{2} \bar{\phi}\right\rangle-\left\langle\Sigma_{2} \bar{\phi}\right\rangle\left\langle\Sigma_{2} \delta \bar{\phi}\right\rangle}{\left\langle\Sigma_{2} \bar{\phi}\right\rangle^{2}} \\
&-\left\langle\theta^{\star}, H \delta \bar{\phi}\right\rangle+\left\langle\delta \theta^{*}, S-H \bar{\phi}\right\rangle \\
&=-\left\langle\delta \theta^{*}, H \bar{\phi}-S\right\rangle-\left\langle H^{*} \theta^{*}, \delta \bar{\phi}\right\rangle \\
&+\left\langle\frac{\Sigma_{1}\left\langle\Sigma_{2} \bar{\phi}\right\rangle-\Sigma_{2}\left\langle\Sigma_{1} \bar{\phi}\right\rangle}{\left\langle\Sigma_{2} \bar{\phi}\right\rangle^{2}}, \delta \bar{\phi}\right\rangle \\
&=-\left\langle\delta \theta^{*}, H \bar{\phi}-S>-\left\langle H^{*} \theta^{*}-G^{-}(\bar{\phi}), \delta \bar{\phi}\right\rangle,\right.
\end{aligned}
$$

where

$$
G^{\prime}(\bar{\phi})=\frac{\Sigma_{1}\left\langle\Sigma_{2} \bar{\phi}\right\rangle-\Sigma_{2}\left\langle\Sigma_{1} \bar{\phi}\right\rangle}{\left\langle\Sigma_{2} \bar{\phi}\right\rangle^{2}} .
$$

To find the stationary functions, we let

$$
\begin{gathered}
\delta E_{1}\left[\bar{\phi}, \theta^{\star}\right]=0=-\left\langle\delta \theta^{\star}, H \bar{\phi}-S\right\rangle- \\
\left\langle H^{\star} \theta^{\star}-G^{-}(\bar{\phi}), \delta \bar{\phi}>,\right.
\end{gathered}
$$

if $\delta \theta^{*}$ and $\delta \bar{\phi}$ are arbitrary values, but not equal to zero, then the requirements for this condition are

$$
H \bar{\phi}_{S}-S=0
$$

and

$$
H^{*} \theta_{S}{ }^{*}-G^{-}\left(\bar{\phi}_{S}\right)=0 .
$$


From Equations (A.9) and (A.2), we have $\bar{\phi}_{S}=\phi$, and from Equations (A.3) and $(A .9)$, the stationary value of the variational functional is

$$
E_{1}\left[\bar{\phi}_{S}, \theta_{S}{ }^{*}\right]=\frac{\left\langle\Sigma_{\eta} \bar{\phi}_{S}\right\rangle}{\left\langle\Sigma_{2} \bar{\phi}_{S}\right\rangle}=W_{n}\left[\bar{\phi}_{S}\right]=W_{n}[\phi] .
$$

Thus, $E_{1}$ is a variational principle for $W_{n}[\phi]$. 


\section{APPENDIX B}

\section{TAYLOR'S SERIES EXPANSION OF $\theta^{*}$}

For a function with three variables, $f(x, y, z)$, the Taylor's series expansion $(41)$ in first order approximation is

$$
\begin{gathered}
f(x, y, z)=f(a, b, c)+f_{x}(a, b, c)(x-a)+f_{y}(a, b, c)(y-b) \\
+f_{z}(a, b, c)(z-c)
\end{gathered}
$$

where $(a, b, c)$ is the position of a given point, $f(a, b, c)$ is the value of the function at the point; $f_{j}(a, b, c), i=x, y, z$ represents the first derivative of the function $f(x, y, z)$ with respect to the variable $i$ at the given point.

Equation (2.19) is a function $\theta^{\star}$ with three variables $\theta^{\star}{ }_{1}$, $\theta^{\star}{ }_{2}$, and $\phi$, we write it here:

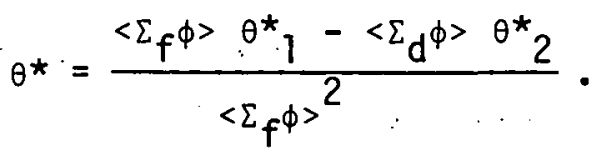

From Equation (B.1), we write the series expansion about a given point as

$$
\theta^{\star} \approx \bar{\theta}^{\star}+\left(\frac{\partial \bar{\theta}^{\star}}{\partial \theta_{1}^{\star}}\right) \delta \theta_{1}^{\star}+\left(\frac{\partial \bar{\theta}^{\star}}{\partial \phi_{2}^{\star}}\right) \delta \theta_{2}^{*}+\left(\frac{\partial \bar{\theta}^{\star}}{\partial \phi}\right) \delta \phi,
$$

where $\bar{\theta}^{\star}$ is given by Equation (2.17), and $\delta \theta^{*} 1$ is $\left(\theta^{*} 1-\bar{\theta}^{*}{ }_{1}\right)$, etc. Substituting Equation (B.2) into Equation (B.3) and carrying out the derivatives, we have 


$$
\begin{aligned}
& \theta^{*}=\bar{\theta} *+\frac{\left\langle\Sigma_{f} \bar{\phi}\right\rangle}{\left\langle\Sigma_{f} \bar{\phi}\right\rangle^{2}} \delta \theta^{\star}{ }_{1} \div \frac{\left\langle\Sigma_{d} \bar{\phi}\right\rangle}{\left\langle\Sigma_{f} \bar{\phi}^{2}\right.} \delta \theta^{*}{ }_{2}+
\end{aligned}
$$

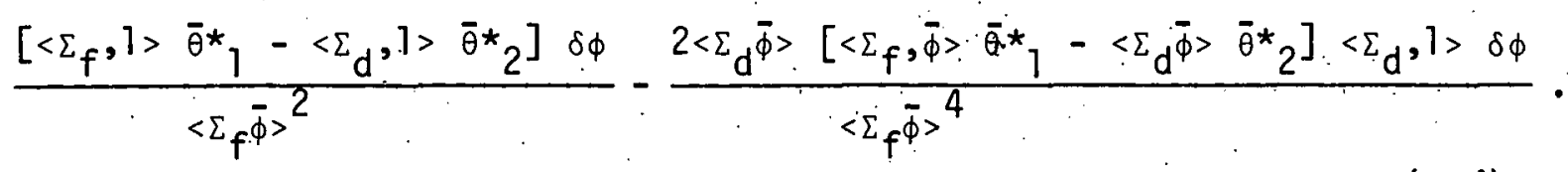

We write Equation (B.3) as

$$
\theta^{*}=\bar{\theta} *+\delta \theta^{\star}
$$

where

$$
\begin{aligned}
& \delta \theta^{*}=\frac{\left\langle\Sigma_{f} \bar{\phi}\right\rangle}{\left\langle\Sigma_{f} \bar{\phi}\right\rangle} \delta \theta^{*}-\frac{\left\langle\Sigma_{d} \bar{\phi}\right\rangle}{\left\langle\Sigma_{f} \bar{\phi}\right\rangle} \delta \theta^{*}+ \\
& \frac{\left[\left\langle\Sigma_{f}, 1\right\rangle \bar{\theta}^{\star}{ }_{1}-\left\langle\Sigma_{d}, 1\right\rangle \bar{\theta}^{\star}{ }_{2}\right]}{\left\langle\Sigma_{f} \bar{\phi}^{2}\right.} \cdot \delta \phi-\frac{2\left\langle\Sigma_{d} \bar{\phi}\right\rangle\left[\left\langle\Sigma_{f}, \bar{\phi}\right\rangle \bar{\theta}^{*}-\left\langle\Sigma_{d}, \bar{\phi}\right\rangle \bar{\theta}^{*}{ }_{2}\right]\left\langle\Sigma_{d}, 1\right\rangle}{\left\langle\Sigma_{f} \bar{\phi}\right\rangle^{4}} \delta \phi .
\end{aligned}
$$

Equation (B.4) shows that $\bar{\theta}^{\star}$ is still a first-order approximation to $\theta^{*}$ if the three variables $\theta^{*}{ }_{1}, \theta^{*}$, and: $\phi$ are in errors of $\delta \theta^{*}{ }_{1}$, $\delta \theta^{*}$, and $\delta \phi$, respectively. 
APPENDIX C

SUPERPOSITION PRINCIPLE FOR A DIFFERENTIAL EQUATION

According to G. A. Korn et al., (16) if there are two solutions, $\phi_{1}(x)$ and $\phi_{2}(x)$, satisfying the following respective linear differential equations

$$
\begin{aligned}
& H_{\phi_{1}}(x)=f_{1}(x), \\
& H_{\phi_{2}}(c)=f_{2}(x),
\end{aligned}
$$

with identical homogeneous linear boundary conditions

then

$$
B \phi(x)=0,
$$

$$
\phi(x)=a \phi_{1}(x)+b \phi_{2}(x),
$$

satisfies the differential equation

$$
H_{\phi}(x)=f(x) \text {, }
$$

where

$$
f(x)=a f_{1}(x)+b f_{2}(x)
$$

where $a$ and $b$ are constant coefficiences.

The adjoint fiux $\bar{\theta}^{*}$ at a reference state for Equation (2.3) is solved by the following equation:

$$
\bar{H}^{\star} \bar{\theta}^{\star}=\frac{\bar{\Sigma}_{d}\left\langle\bar{\Sigma}_{f} \bar{\phi}\right\rangle-\bar{\Sigma}_{f}\left\langle\bar{\Sigma}_{d} \bar{\phi}\right\rangle}{\left\langle\bar{\Sigma}_{f} \bar{\phi}\right\rangle^{2}} .
$$


Rearranging the right hand side of Equation (C.6) leads to

$$
\bar{H} \star \bar{\theta}^{\star}=\bar{\Sigma}_{d}\left(\frac{1}{\left\langle\bar{\Sigma}_{f} \bar{\phi}\right\rangle}\right)-\bar{\Sigma}_{f}\left(\frac{\left\langle\bar{\Sigma}_{d} \bar{\phi}\right\rangle}{\left\langle\bar{\Sigma}_{f} \bar{\phi}^{2}\right.}\right)
$$

We compare Equation (C.7:) with Equation (C.5), and specify

that

$$
a=\frac{1}{\left\langle\bar{\Sigma}_{f} \bar{\phi}\right\rangle} \text {, }
$$

and

$$
\mathrm{b}=-\frac{\left\langle\bar{\Sigma}_{\mathrm{d}} \bar{\phi}\right\rangle}{\left\langle\bar{\Sigma}_{\mathrm{f}} \bar{\phi}\right\rangle} .
$$

Then

$$
\begin{gathered}
\bar{\theta}^{\star}=\mathrm{a} \bar{\theta}^{\star}{ }_{1}+\mathrm{b} \bar{\theta}^{\star}{ }_{2} \\
=\left(\frac{1}{\left\langle\bar{\Sigma}_{f} \bar{\phi}\right\rangle}\right) \bar{\theta}^{\star}{ }_{1}-\left(\frac{\left\langle\bar{\Sigma}_{\mathrm{d}} \bar{\phi}\right\rangle}{\left\langle\bar{\Sigma}_{f} \bar{\phi}\right\rangle}\right) \bar{\theta}^{\star}{ }_{2},
\end{gathered}
$$

where $\bar{\theta}^{\star}$, and $\bar{\theta}^{\star}{ }_{2}$ are solutions of the differential equations:

$$
\bar{H} *_{\theta}{ }_{1}=\dot{\bar{\Sigma}}_{\mathrm{d}},
$$

and

$$
\bar{H} \bar{\theta}^{*}{ }_{2}=\bar{\Sigma}_{f} .
$$




\section{APPENDIX D}

DISCRETE FORM OF THE VARIATIONAL FUNCTIONAL FOR SLAB GEOMETRY

From Equation (3.18),

$$
\mathrm{F}_{\mathbf{i}}\left[\bar{\phi}, \bar{\theta}^{*}{ }_{i}\right]=\left\langle\Sigma_{\mathbf{j}} \bar{\phi}\right\rangle+\left\langle\bar{\theta}^{\star}{ }_{j}, \Delta S-\Delta H \bar{\phi}\right\rangle,
$$

where $\Delta H \bar{\phi}$ for slab: geometry is given by

$$
\begin{aligned}
& \Delta H(X, E, \mu) \bar{\phi}(x, E, \mu)=\Delta \Sigma(X, E) \bar{\phi}(X, E, \mu)-\frac{X(E)}{4 \pi} \& s \Delta\left(\nu \Sigma_{f}\left(X, E^{\prime}\right) \cdot \bar{\phi}\left(x, E^{\prime}, \mu^{\prime}\right)\right. \\
& \text { - } \mathrm{dE}^{-} \mathrm{d} \bar{\Omega}^{-}-\iint \Delta \Sigma_{S^{\prime}}\left(\mathrm{x} ; \mathrm{E}^{-}, \bar{\Omega}^{-} \rightarrow \mathrm{E}, \bar{\Omega}\right) \bar{\phi}\left(\mathrm{x}, \mathrm{E}^{-}, \mu^{-}\right) \mathrm{dE}^{-} \mathrm{d} \bar{\Omega}^{-},
\end{aligned}
$$

we have, after integration over energy interval

$$
\begin{aligned}
& F_{i}\left[\bar{\phi}_{g}, \bar{\theta}^{*}{ }_{i g}\right]=\sum_{g=1}^{G} \int \delta \Sigma_{i g}(x, \mu) \cdot \bar{\phi}_{g}(x, \mu) d x d \mu+ \\
& \sum_{g=1}^{G} \int \theta_{i \cdot g}^{-*}(x, \mu) \Delta S_{g}(x, \mu) d x d \mu- \\
& \sum_{g=1}^{G} \int \delta \bar{\theta}^{\star}{ }_{i g}(x, \mu) \Delta \Sigma_{g}(x) \bar{\phi}_{g}(x, \mu) d x d \mu+ \\
& \sum_{g=1}^{G} \frac{x_{g}}{4 \pi} \int \delta\left[\bar{\theta}_{i g}^{*}(x, \mu) \sum_{g^{-=1}}^{G} \Delta\left(\nu \Sigma_{f}(x)\right) g^{-} \int \bar{\phi}_{g^{-}}\left(x, \mu^{-}\right) d \bar{\Omega}^{-}\right] d x d \mu+
\end{aligned}
$$

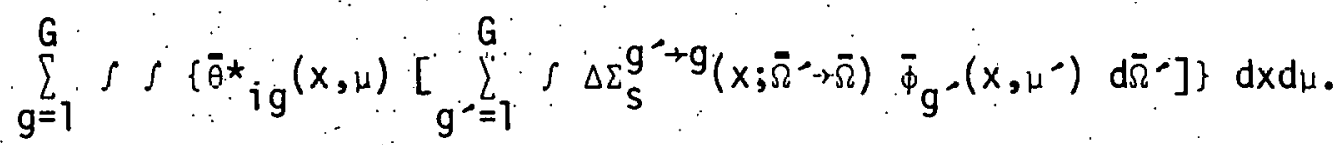


The scattering cross section as well as angular fluxes are expanded in the following Legendre polynomals ${ }^{(3)}$

$$
\begin{aligned}
& \Delta \Sigma_{S}^{g^{\prime \prime} \rightarrow g}\left(x ; \bar{\Omega}^{-} \rightarrow \bar{\Omega}\right)=\Delta \Sigma_{S}^{g^{\prime} \rightarrow g}\left(x ; \mu_{0}\right) \\
& =\sum_{n=0 /}^{\text {ISCT }}\left(\frac{i}{2 \pi}\right) \Delta \Sigma_{s n}^{g^{\prime} \rightarrow g}(x) P_{n}\left(\mu_{0}\right), \\
& \bar{\theta}^{\star}{ }_{i g}(x, \mu)=\sum_{n=0}^{I S C T}\left(\frac{2 n+1}{2}\right) \cdot \bar{\theta}^{\star}{ }_{i g n}(x) P_{n}(\mu),
\end{aligned}
$$

and

$$
\bar{\phi}_{g}(x, \mu)=\sum_{n=0}^{\text {ISCT }}\left(\frac{2 n+1}{2}\right) \bar{\phi}_{g n}(x) P_{n}(\mu),
$$

where

$$
\begin{gathered}
\Delta \Sigma_{s n}^{g^{\circ} \rightarrow g}(x)=2 \pi\left(\frac{2 n+1}{2}\right) \int_{-1}^{1} \cdot \Delta \Sigma_{s}^{g^{\circ} \rightarrow g}\left(x ; \mu_{0}\right) P_{n}\left(\mu_{0}\right) d \mu_{j}^{\prime} ; \\
P_{n}\left(\mu_{0}\right)=P_{n}(\mu) P_{n}\left(\mu^{\prime}\right)+2 \sum_{m=1}^{n} \frac{(n-m) !}{(n+m) !} P_{n}^{m}(\mu) P_{n}^{m}\left(\mu^{-}\right) \cos m\left(\Phi-\Phi^{-}\right) \\
\bar{\theta}_{i g n}^{*}(x)=\int_{-1}^{1} \bar{\theta}_{i g}^{*}(x, \mu) P_{n}(\mu) d \mu,
\end{gathered}
$$

and

$$
\bar{\phi}_{g n}(x)=\int_{-1}^{1} \bar{\phi}_{g}(x, \mu) P_{n}(\mu) d \mu .
$$

After substituting Equations (D.4) through (D.10) into Equation (D.3) and making use of the mechanical quadrature for the integration of direction cosine $\mu$, we have 


$$
\begin{aligned}
& F_{i}\left[\bar{\phi}_{g}, \bar{\theta}_{i g}^{*}\right]=\sum_{g=1}^{G} \sum_{j=1}^{I} V_{j} \Sigma_{i g}\left(x_{j}\right) \bar{\phi}_{g}\left(x_{j}\right)+\sum_{g=1 j=1}^{G} \sum_{j=1}^{I} V_{j} \sum_{K=1}^{N O A} \bar{\theta}_{i g}^{*}\left(x_{j}, \mu_{K}\right) \\
& \text { - } \Delta S_{g}\left(x_{j}, \mu_{K}\right) w_{K}-\sum_{g=1}^{G} \cdot \sum_{j=1}^{I} v_{j} \Delta \Sigma_{g}\left(x_{j}\right) \sum_{K=1}^{N O A} \bar{\theta}_{i g}\left(x_{j}, \mu_{K}\right) \bar{\phi}_{g}\left(x_{j}, \mu_{K}\right) w_{K} \\
& +\sum_{g=1}^{G}\left(\frac{X_{g}}{2}\right) \sum_{j=1}^{I} v_{j} \bar{\theta}^{*}{ }_{i g}\left(x_{j}\right)\left[\sum_{g=1}^{G} \Delta\left(v \Sigma_{f}\left(x_{j}\right)\right) g^{-}-\bar{\phi}_{g} \cdot\left(x_{j}\right)\right]+ \\
& \sum_{g=1}^{G} \sum_{j=1}^{I} V_{j} \sum_{n=0}^{I S C T} \bar{\theta}^{\star}{ }_{i g n}\left(x_{j}\right) \sum_{g=1}^{G} \Delta \Sigma_{s n}^{g^{\prime} \rightarrow g}\left(x_{j}\right) \phi_{g:-n}\left(x_{j}\right),
\end{aligned}
$$

where NOA is the total number of angles, $I$ is the total number of intervals, $W_{K}$ is the weighting factor for the $K^{\text {th }}$ angle, and all other terms are defined in Section C of Chapter III, page 30. 


\begin{abstract}
APPENDIX E
CALCULATION OF $<\bar{\theta}^{\star}, S>$ FOR ONE-DIMENSIONAL PROBLEM
\end{abstract}

A. Introduction

The purpose of this appendix is to describe the program used to calculate the parameter $\left\langle\bar{\theta}^{\star}, S\right\rangle$ for one-dimensional problem. A description of input and the code listing are also included.

\title{
B. Program Description
}

The numerical formulation for the integral parameter $\langle\bar{\theta} \star, S\rangle$ is

$$
\begin{gathered}
\left\langle\bar{\theta}^{\star}, S\right\rangle=\iint \bar{\theta}^{\star}(r, E) S(r, E) d E d r \\
=\sum_{g=1}^{G} \sum_{J=1}^{I} \overline{0}_{g}^{\star}(J) s_{g}(J) \Delta x_{j}
\end{gathered}
$$

where

$$
\begin{aligned}
G & =\text { total neutron energy group number, } \\
I & =\text { total space interval number, } \\
\Delta X_{J} & =\text { the size of the } J^{\text {th }} \text { interval, } \\
S_{j}(J) & =x_{g} S(J), \\
\bar{\theta}^{*}(J) & =\text { the adjoint flux at } g^{\text {th }} \text { group and the } J^{\text {th }} \text { interval. }
\end{aligned}
$$

The integration is carried out by summing the products $\bar{\theta}_{g}^{*}(J) S_{g}(J)$ over the energy-groups and the space-intervals. The adjoint flux $\bar{\theta}^{*}{ }_{g}(J)$ is calculated by. ANISN code, ${ }^{(10)}$ and the fixed source for 
each interval $S(J)$ is specified by the way we solve the flux equation

$$
H_{\phi}=S \text {. }
$$

The specification of interval size should be the same as that for calculation of the adjoint flux. We calculate the interval size by giving the zone boundaries. The code is written for 50-group, 18-zone, and a total of 102 intervals.

\section{Input Cards}

Card 1 (20A4)

Title Card.

Card $26(3 x, F 9.0,8 x)$

$I=1,50$

$(\operatorname{PHI}(I, J), J=1,102)$ Adjoint scalar flux of each group.

Card $3(7 E 11.5)$

$\mathrm{CHI}$

Fission spectrum of each group, start from group 1 .

Card $4(6 \mathrm{~F} 10.6)$

SN

Source distribution of each core zone.

Card 5 (6F 12.5$)$ 
D. Listing of the Code

C CAL OF PHI*S*V IN 1-D

DIMENSION PHI $(50,102), S(50,102), R(103)$,

LA(20), CHI(50),SN(12), ZR (18)

$\operatorname{READ}(5,2)$ A

2 FORMAT (20A4)

WRITE $(6,1) \quad(A(I), I=1,20)$

1 FORMAT $(10 \cdot, 4 x ; 20 A 4)$

DO $71=1,50$

$7 \operatorname{READ}(5,10, E N D=200)(\operatorname{PHI}(I, J), J=1,102)$

10 FORMAT $(613 X, F 9.0), 8 X)$

WRITE $(6,81)$

81 FORMATI20X, "FLUX'/1

$J 1=0$

Do $90 \quad$ I $1=1.49,8$

$\mathrm{Jl}=\mathrm{Jl}+8$

$I F(I \perp-E Q .49) \quad J I=50$

WRITE $(6,80)((P H I(I, J), I=I 1, J)), J=1,102 ; 10)$

80 FORMA T $(10 X, 8 E 12.5)$

SO CONT I NUE

C READ SOURCES

200 DO $30 \quad I=1,50$

DO 3:J $\mathrm{J}=1,102$

$30 \mathrm{~S}(I ; J)=0$.

READ $(5,25) \mathrm{CHI}$

25 FORMAT (7E 11.5$)$

WRITE $(6,27) \mathrm{CHI}$

27 FORMATI//4X, 'CHI $/ / 50(/ 4 X, 1$ PE 12.5$))$

READ $(5,50) S N$

50 FORMAT $(6 F 10.6)$

WRI TE $(6,55)$ SN

55 FORHAT $(/ / 4 X, \cdot S N \cdot / / 12(/ 4 X, F 10.6))$

DO $60 \quad I=1,12$

$L=22+(I-1) * 5$

$L P L=L+1$

$L L L=L+4$

DO $40 \mathrm{~J}=1,50$

$S(J, L)=C H I(J) * S N(I)$

DD 45 II I $=L P I, L L 1$

$45 \mathrm{~S}(\mathrm{~J}, 1 \mathrm{I}(1)=\mathrm{S}(\mathrm{J}, \mathrm{L})$

40 CONTINUE

tO CONTINUE

C READ ZONE BOUNDERIES

$R E A D(5,70)$ 2R

70 FORMA T (6F12.5)

WRITE $(6,66)$ ZR

66 FORMAT (//4X, $Z R ! / / 181 / 4 X, F 12.5)$ )

C RADIUS FOR INTERVALS

$R(1)=0$.

$R(8)=Z R(1)$

DO $77 \quad I I=1,3$

$I I=(I I-1) * 7+2$ 


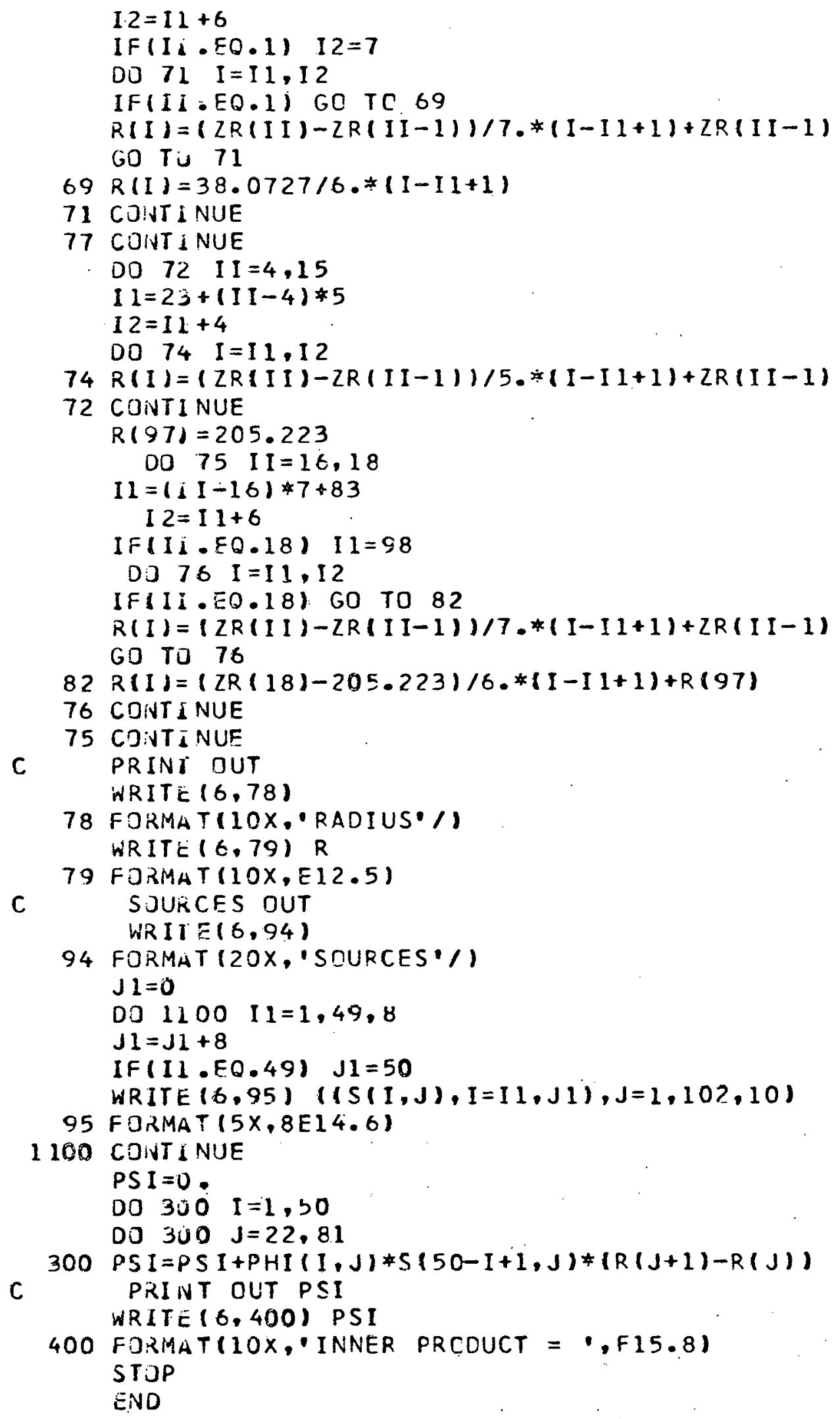




\section{APPENDIX $F$}

CALCULATION OF $<\bar{\theta} \star$,S $>$ FOR TWO-DIMENSIONAL PROBLEM

\section{A. Introduction}

The objectives of this appendix are: (a) description of the routine used to calculate the value of $\langle\bar{\theta} \star, S\rangle$ for two-dimensional problem and (b) present a description of input and the program listing.

\section{B. Program Description}

The numerical formula for the parameter $\langle\bar{\theta} *, S\rangle$ is explicitly written as:

$$
\begin{gathered}
\langle\bar{\theta} \star S\rangle=\iint \bar{\theta} \star(\bar{r}, E) S(\bar{r}, E) d E d \bar{r} \\
=\sum_{g=1}^{G} \sum_{i=1}^{I m a x} \sum_{j=1}^{J \max } \bar{\theta}_{g}{ }_{g}(i, j) S_{g}(i, j) \Delta x_{i} \Delta y_{j},
\end{gathered}
$$

where

$$
\begin{aligned}
\operatorname{Imax} & =\text { the total number of } \text { radial }^{\mathrm{a}} \text { interval. } \\
J \max & =\text { the total number of } \text { axial }^{\mathrm{b}} \text { interval } \\
(i, j) & =\text { the interval at the }(i, j) \text { position, } \\
\Delta x_{i}= & \text { the size of the } i^{\text {th }} \text { interval in the axial direction, and } \\
\Delta y_{j}= & \text { the size of the } j^{\text {th }} \text { interval in the axial direction, and } \\
& \text { others are specified in Appendix } E .
\end{aligned}
$$

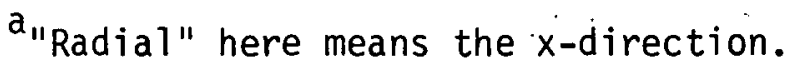

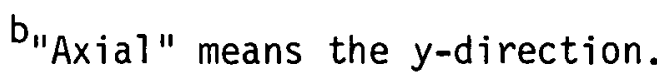


At the time of this calculation, $G$ was 14 , Imax was 43 , and Jmax was $41 . \bar{\theta}^{*}(i, j)$ was calculated by the DOT code, ${ }^{(31)}$ and input by tape for this computation. The specifications of the fixed source and the interval size are the same as used for DOT. Since the source is zero outside the fueled region of the reactor, the program makes the interval summation over core zones only.

\section{Input Cards}

Card 1 (7E11.5)

$\mathrm{CHI}$ Fission spectrum of each group, start from group number 1 . (Source in fueled zone only.)

Card 2 (7E11.5)

$$
\begin{array}{ll}
J=9,29 & \text { Fixed source distribution for } \\
(S(I, J), I=12,34) & \text { each interval. }
\end{array}
$$

Card $3(15 A 4$, I2)

TITLE

NFLSV
Title for the run. Logical number of the flux gape. 
D. Listing of the Code

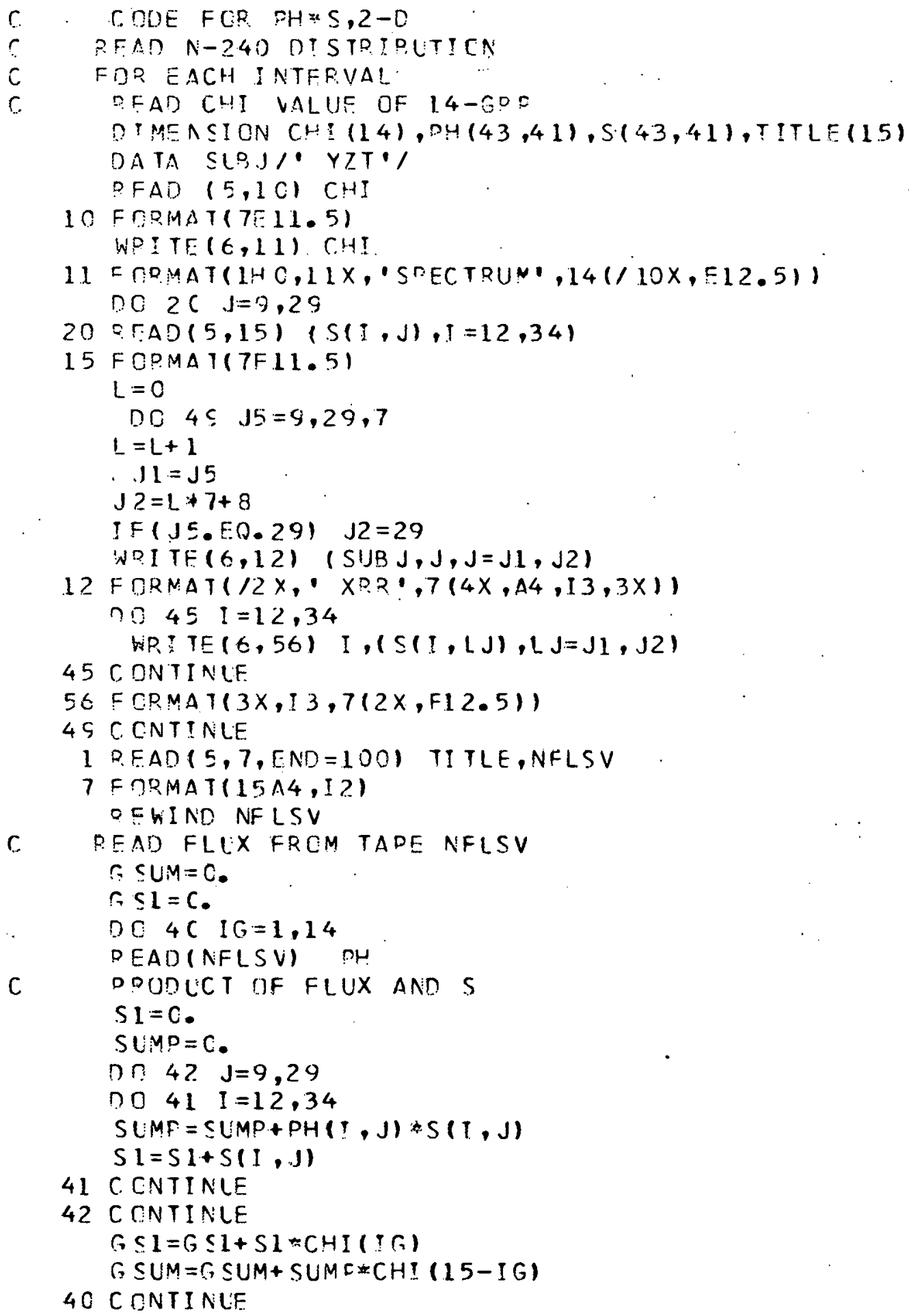




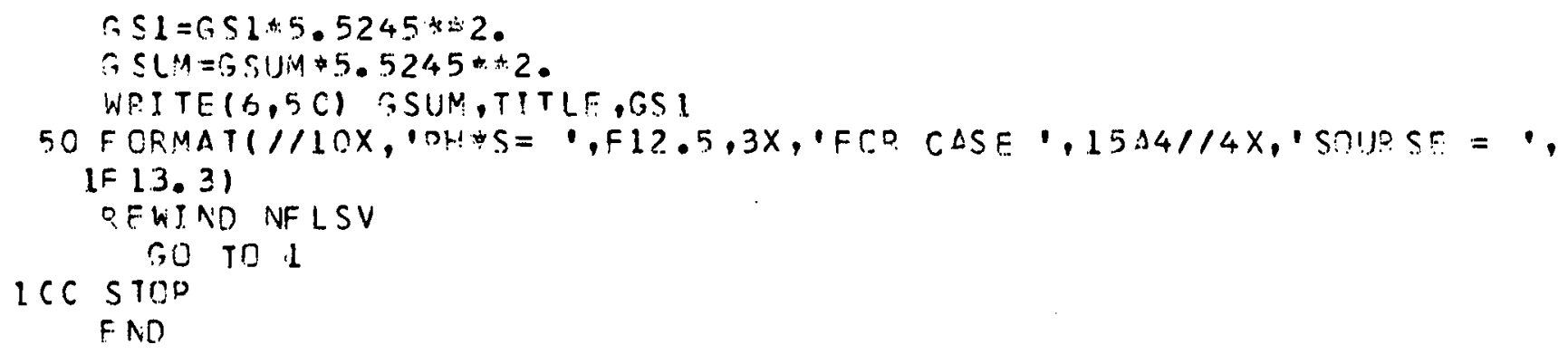

FND 
THIS PAGE

WAS INTENTIONALLY

LEFT BLANK 
ORNL/TM-5239

UC -79d - LMFBR Physics

INTERNAL DISTRIBUTION

1-3. L. S. Abbott

4. J. W. Allen

5. R. G. Alsmiller

6. J. D. Amburgey

7. J. Barish

8. D. E. Bartine

9. V. C. Baker

10. J. A. Bucholz

11. T. J. Burns

12. R. L. Childs

13. C. E. Clifford

14. S. N. Cramer

15. N. F. Cross

16. G. W. Cunningham

17. J. D. Drischler

18. M. B. Emmett

19. W. W. Engle, Jr.

20-29. G. F. Flanagan

30. W. E. Ford

31. V. M. Forsberg

32. T. B. Fowler

33. T. A. Gabriel

34. N. M. Greene

35. W. 0. Harms

36. T. J. Hoffman

37. J. D. Jenkins

38. T. W. Kerlin

39. R. A. Lillie

40. J. L. Lucius

41. J. W. McAdoo

42. F. C. Maienschein

43. J. T. Mihalczo

44. J. E. Mott

45. F. R. Mynatt

46. E. M. Oblow

47. Y. M. Ozal

48. J. V. Pace

49. A. M. Perry
50. L. M. Petrie

51. H. Postma

52. K. R. Piety

53. W. A. Rhoades

54-58. J. C. Robinson

59. R. T. Santoro

60. R. W. Roussin

61. D. L. Selby

62. F. S. Shahrokhi

63. D. B. Simpson

64. C. 0. Slater

65. P. N. Stevens

66. J. S. Tang

67. M. L. Tobias

68. E. T. Toml inson

69. D. L. Trauger

70. K. R. Turnbul1

71. D. R. Vondy

72. C. R. Weisbin

73. R. M. Westfall

74. J. E. White

75. G. E. Whitesides

76. L. R. Williams

77. M. L. Williams

78. R. Q. Wright

79. A. Zucker

80. P. F. Fox (consultant)

81. W. W. Havens (consultant)

82. A. F. Henry (consultant)

83. R. E. Uhrig (consultant)

84-85. Central Research Library

86. $Y-12$ Document Reference Section

87-88. Laboratory Records Department

89. Laboratory Records, ORNL RC

90. RSIC

91. M. L. Tobias

92. H. Goldste in (consultarit)

\section{EXTERNAL DISTRIBUTION}

93-94. Director, Division of Reactor Research and Development, ERDA, Washington, DC 20545

95. Research and Technical Support Division, ERDA-ORO

96. Director, Reactor Division, ERDA-ORO

97-300. For distribution as shown in TID-4500, Distribution Category, UC-79d, Liquid Metal Fast Breeder Reactor Physics 